\title{
NMR Studies on [2 + 3] Cycloaddition of Nitrile Oxides to Polyunsaturated Medium Size Rings
}

\author{
Mirostaw Gucma, W. Marek Gotębiewski* and Alicja K. Michalczyk \\ Institute of Industrial Organic Chemistry, Annopol 6, 3-236 Warsaw, Poland
}

\begin{abstract}
Site selectivity, regioselectivity and stereoselectivity of $[2+3]$ cycloaddition of 4-trifluoromethylbenzonitrile oxide to polyunsaturated medium size rings including 1,5,9-cyclododecatriene, 11-membered sesquiterpenes, 1,3-cyclooctadiene and 5-vinyl-2norbornene were examined. Site selectivity was correlated with electron charges of alkenyl carbon atoms. Structure of the products has been established by an extensive application of 1D and 2D ${ }^{1} \mathrm{H}$ and ${ }^{13} \mathrm{C}$ nuclear magnetic resonance (NMR) spectroscopy and electrospray ionization mass spectrometry. Some of the obtained products showed moderate fungicidal activities.
\end{abstract}

Keywords: [2 + 3] dipolar cycloaddition, sesquiterpenes, site selectivity, 2D NMR, fungicides

\section{Introduction}

Isoxazolines are one of major classes of five-membered nitrogen containing heterocycles, found in a large number of natural products and biologically active compounds. A variety of synthetic methods has been elaborated for preparation of 2-isoxazolines, of which the most convenient and attractive route is the $[2+3]$ dipolar cycloaddition of nitrile oxides to alkenes. ${ }^{1}$ 2-Isoxazolines can be easily reduced to several synthetically important compounds such as $\beta$-hydroxy ketones, $\beta$-hydroxy esters, $\alpha, \beta$-unsaturated carbonyl compounds or iminoketones. ${ }^{2}$

The nitrile oxides can be formed either by Huisgen method from aldoximes by chlorination and base-induced dehydrochlorination ${ }^{1}$ or by dehydration of primary nitro compounds by phenyl isocyanates (Mukayama method) ${ }^{3}$ or ethyl chloroformate (Shimizu method). ${ }^{4}$

A key feature of the cycloaddition is the cis-stereospecificity - from $E$-alkenes 4,5-anti isomers are produced and from $Z$-alkenes 4,5 -syn products are obtained. ${ }^{5}$ Reactions of monosubstituted and 1,1-disubstituted alkenes are very regioselective favoring strongly 5 -substituted 2-isoxazolines. On the other hand, 1,2-disubstituted olefins usually afford mixtures of regio- and stereoisomers. Two methods have been used to solve these problems. One approach was a substrate control, an application of appropriately functionalized cycloaddends. The second more effective approach relied on metal complexes acting

*e-mail: golebiewski@ipo.waw.pl as catalysts or on organocatalysts. Shortage of reports on metal assisted 1,3-dipolar cycloadditions of nitrile oxides was due to interference of catalyst with generation of these dipoles and formation of unreactive complexes. ${ }^{6}$

Site-selectivity of nitrile oxides cycloaddition to polyunsaturated alkenes was examined in several laboratories. In reactions of nitrile oxides with dimethyl 7-(diphenylmethylene)bicyclo[2.2.1]hept-2-ene-5,6dicarboxylate only disubstituted norbornene double bond participated. ${ }^{7}$ Similarly, in recently examined cycloadditions of aryl nitrile oxides to norbornenes substituted with an acrylate-derived moiety, only adducts to norbornene system were formed with good site and exo selectivity. ${ }^{8}$ Site selectivity and regioselectivity of cycloaddition to cyclohexene derivatives was studied in our group. ${ }^{9}$

2,6-Dichlorobenzonitrile oxide reacts with isothiazolones at the ethylenic double bond. Mesitonitrile oxide, on the other hand, adds preferentially to the carbonyl double bond. ${ }^{10}$

No $[2+3]$ cycloaddition reactions of nitrile oxides to medium ring cycloalkenes was reported before, to the best of our knowledge, presumably because of the low reactivity of these dipolarophiles. However, other types of cycloadditions to these systems are known. Diels-Alder cycloaddition of benzotropolone with humulene (11-membered sesquiterpene) was described. ${ }^{11}$ Acetylacetonatoboron difluoride and oxalate undergo cycloaddition from their singlet excited state with cyclic olefins to give non-regiospecific products. A conjugated diene, 1,3-cyclooctadiene, 
reacted similarly but with slower rates. ${ }^{12}$ Singlet excited dibenzoyl(methanato)boron difluoride underwent also cycloaddition to 1,3 -cyclooctadiene..$^{13}$ Reaction of $\mathrm{SO}$ with 1,3-cyclooctadiene gave the corresponding [1+4] adduct in low $2 \%$ yield. ${ }^{14} 1,3$-Cyclooctadiene was unreactive in titanium complex catalyzed $[2+6]$ cycloaddition reaction with cycloheptatriene..$^{15}$ Reaction of unconjugated triene 1,5,9-cyclododecatriene with chlorosulfonyl isocyanate (CSI) gave an unsaturated $\beta$-lactam product, where only one double bond had reacted..$^{16}$ Acetylacetone underwent a photochemical cycloaddition to 1,5,9-cyclododecatriene (the de Mayo reaction). ${ }^{17}$ The sterically controlled cycloaddition of dichloroketene to unreactive olefin, 5-methylene-2-norbornene, has been accomplished. ${ }^{18}$ It resulted in a spirocyclobutanone derivative formed by addition across the exocyclic double bond.

The sesquiterpenes possess fifteen carbons, derived from three isoprenoid units. This is a vast group of naturally occurring substances, containing an immense range of structural diversity which includes acyclic, monocyclic, bicyclic, tricyclic and tetracyclic compounds. Many sesquiterpenes display biological activity including antimicrobial, antitumor and cytotoxic properties. ${ }^{19}$ Some bisnor-sesquiterpenes exhibit allelopathic activity. ${ }^{20}$

Herein we present results of our research concerning site selectivity, regioselectivity and stereoselectivity of 1,3-dipolar cycloaddition of 4-trifluoromethylbenzonitrile oxide to polyunsaturated medium rings including 11 -membered sesquiterpenes $\mathbf{5}, \mathbf{8}$ and $\mathbf{1 0}$, 1,5,9-cyclododecatriene (13), 1,3-cyclooctadiene (18) and 5-vinyl-2-norbornene (21) envisaging to obtain new biologically active compounds. Structure of the products has been established by an extensive application of $1 \mathrm{D}$ and $2 \mathrm{D}{ }^{1} \mathrm{H}$ and ${ }^{13} \mathrm{C}$ nuclear magnetic resonance (NMR) spectroscopy and electrospray ionization mass spectrometry (ESI-MS).

\section{Experimental}

\section{Materials and physical measurements}

Reagent-grade chemicals were used without further purification unless otherwise noted. Compounds 5, 8, 10, 13, 18, 21 were purchased from Aldrich. Hydroximinoyl acid chlorides were prepared from the corresponding aryl aldehyde oximes and $N$-chlorosuccinimide (NCS) in $N, N$-dimethylformamide (DMF). ${ }^{21}$

Spectra were recorded as follows: Fourier transform (FT) infrared (IR) spectra on a JASCO FTIR-420 spectrometer; ${ }^{1} \mathrm{H},{ }^{13} \mathrm{C}$ NMR, 2D correlation spectroscopy (COSY), 2D heteronuclear single quantum coherence (HSQC), 2D heteronuclear multiple bond correlation (HMBC) and 2D rotating frame nuclear Overhauser effect spectroscopy (ROESY) analyses on a Bruker AVANCE III $500 \mathrm{MHz}$ and a Varian VNMRS 600 spectrometers in deuterated chloroform. The ${ }^{1} \mathrm{H}$ NMR spectra were recorded using single-pulse sequence and spectral width (SW) of $10000 \mathrm{~Hz}, 30^{\circ}$ pulse width (pw) of $9.7 \mu \mathrm{s}$, an acquisition time (at) of ca. $3.3 \mathrm{~s}$ and $64 \mathrm{k}$ complex points. The free induction decays (FIDs) were processed with zero filling. The ${ }^{13} \mathrm{C}$ spectra were obtained using a spectral range of ca. $31250 \mathrm{~Hz}, 30^{\circ}$ pulse width (3.3 $\mu \mathrm{s})$, an acquisition time of ca. $1.05 \mathrm{~s}$, a relaxation delay of $1.5 \mathrm{~s}$ and collecting $64 \mathrm{k}$ complex points. Chemical shifts are given in $\operatorname{ppm}(\delta)$ relative to tetramethylsilane (TMS) as an internal standard and coupling constants are reported in $\mathrm{Hz}$. The 2D gradient selected (g)COSY and ROESY spectra were run using spectral width (ca. $6000 \mathrm{~Hz}$ ) in both dimensions, at $=0.18 \mathrm{~s}, 2-4$ (COSY) and 4-8 (ROESY) transients per 512 increments, relaxation delay (d1) of 2.0 s. Prior to FT the data were processed using squared sinebell (COSY) and gaussian (ROESY) multiple function. In the case of the ROESY experiments spinlock time ca. $200 \mathrm{~ms}$ was chosen. The echo-antiecho phase-sensitive ${ }^{1} \mathrm{H} /{ }^{13} \mathrm{C}$ gHSQC correlation were obtained with an at of $0.18 \mathrm{~s}$, spectral window of $5500 \mathrm{~Hz}$ (F2) and $25500 \mathrm{~Hz}(\mathrm{~F} 1), 512$ increments in the ${ }^{13} \mathrm{C}$ dimension, $\mathrm{d} 1=2.0 \mathrm{~s}$ and 2 transients per $\mathrm{t}_{1}$ increment. Experiments were optimized for ${ }^{1} J(\mathrm{C}-\mathrm{H})=145 \mathrm{~Hz}$. The data were zero-filled to 2048 points and processed using cosine-squared window function in both dimensions prior to Fourier transformation. The proton and carbon $90^{\circ}$ pulse lengths were 8 and $15 \mathrm{~ms}$, respectively. The ${ }^{1} \mathrm{H}-{ }^{13} \mathrm{C}$ HMBC experiments with pulse field gradient (PFG) coherence selection using two PFG pulses were recorded with the following parameters: an at of $0.18 \mathrm{~s}$, spectral windows of $5500 \mathrm{~Hz}$ (F2) and $25500 \mathrm{~Hz}(\mathrm{~F} 1), 512$ increments in the ${ }^{13} \mathrm{C}$ dimension, 4 transients per increment and $\mathrm{d} 1$ of $2 \mathrm{~s}$. This kind of experiment was optimized for ${ }^{\mathrm{n}} \mathrm{J}(\mathrm{C}-\mathrm{H})=10 \mathrm{~Hz}$. The proton and carbon $90^{\circ}$ pulse lengths were 8 and $15 \mathrm{~ms}$, respectively. The ${ }^{1} \mathrm{H}-{ }^{15} \mathrm{~N}$ HMBC experiments with PFG coherence selection using two PFG pulses were recorded with the following parameters: an at of $0.25 \mathrm{~s}$, spectral windows of $6500 \mathrm{~Hz}$ (F2) and $12000 \mathrm{~Hz}$ (F1); $2 \times 256$ increments in the ${ }^{15} \mathrm{~N}$ dimension, 16-32 transients per increment and $\mathrm{d} 1$ of $1.5 \mathrm{~s}$, optimized for ${ }^{\mathrm{n}} J(\mathrm{~N}-\mathrm{H})=5.0 \mathrm{~Hz}$. The proton and nitrogen $90^{\circ}$ pulse lengths were 7.2 and $30.0 \mathrm{~ms}$, respectively.

Electron ionization (EI) MS were run on an AMD M-40 instrument, ESI-MS on an LCT (Micromass) apparatus. Flash chromatography was carried out using silica gel S 230-400 mesh (Merck) using hexane-ethyl acetate mixtures as an eluent. Calculations of electron charges on alkenyl carbon atoms, molecular modelling 
and substrate HOMO/LUMO energies were calculated with the Hyperchem 7.5 program using semiempirical AM1 method. ${ }^{22}$

Cycloaddition reaction of dipolarophiles 5, 8, 10, 13, 18 and 21 with 4-trifluoromethylbenzonitrile oxide (4). A general procedure for preparation of $6,7,9,11,12,14-17,19,20$, and 22-27

4-Trifluoromethylbenzonitrile oxide (4) was generated as follows: a solution of the corresponding chloroxime $(0.25 \mathrm{~g}$, $1.12 \mathrm{mmol})$ in dry dichloromethane was passed through an Amberlyst-21 column and added dropwise over $30 \mathrm{~min}$ to the solution of a dipolarophile in dry dichloromethane, and the solution was stirred overnight at room temperature. Water was added, organic layer was separated and the aqueous one extracted with dichloromethane. The combined organic layers were dried $\left(\mathrm{MgSO}_{4}\right)$ and the product was purified by flash column chromatography.

3a,12a-trans-(6E,10E)-6,6,9,12a-Tetramethyl-3-[4(trifluoromethyl)phenyl]-3aH, $4 \mathrm{H}, 5 \mathrm{H}, 8 \mathrm{H}, 9 \mathrm{H}, 12 \mathrm{H}, 12 \mathrm{a} H$ cycloundeca[d][1,2]oxazole (6)

Celadon-yellow wax; yield: 35\%; ${ }^{1} \mathrm{H}$ NMR $(500 \mathrm{MHz}$, $\left.\mathrm{CDCl}_{3}\right) \delta 7.73$ (d, 2H, J 8.3 Hz, H2', H6'), 7.67 (d, 2H, $J$ J $8.3 \mathrm{~Hz}, \mathrm{H} 3$ ', H5'), 5.24 (ddd, 1H, $J$ 16.5, 10.0, $5.0 \mathrm{~Hz}$, H4), 5.18 (d, 1H, J $16.5 \mathrm{~Hz}, \mathrm{H} 5), 4.97$ (bd, $1 \mathrm{H}, J 11.8 \mathrm{~Hz}$, H8), 3.37 (dd, 1H, $J 11.0,2.0 \mathrm{~Hz}, \mathrm{H} 1$ ), 2.32 (dq, 1H, $J 11.8$, $5.0 \mathrm{~Hz}, \mathrm{H} 3 \mathrm{a}$ ), 2.20 (td, 1H, J 12.5, 5.0 Hz, H10a), 2.16-2.12 (m, 1H, H7a), 2.14-2.10 (m, 2H, H6b, H10b), 1.88 (d, 1H, $J 12.5 \mathrm{~Hz}, \mathrm{H} 7 \mathrm{~b}$ ), 1.70-1.63 (m, 1H, H11a), 1.62 (s, 3H, H16), 1.61-1.54 (m, 1H, H11b), 1.53 (s, 3H, H13), 1.17 (s, 3H, H15), 1.15 (s, 3H, H14); ${ }^{13} \mathrm{C}$ NMR (125.9 MHz, $\left.\mathrm{CDCl}_{3}\right) \delta$ 160.4 (C12), 143.4 (C5), 133.8 (C1'), 133.0 (C4), 131.4 (q, J 32.5 Hz, C4'), 131.1 (C9), 128.5 (C5), 127.4 (C6', C2'), 125.7 (q, J 3.9 Hz, C5', C3'), 123.9 (q, J 265.2 Hz, F ${ }_{3}$ ), 122.2 (C4), 90.8 (C2), 47.2 (C1), 42.8 (C3), 39.5 (C7), 37.7 (C6), 37.0 (C10), 30.2 (C15), 24.5 (C14), 23.9 (C11), 19.3 (C13), $15.8(\mathrm{C} 16) ;{ }^{19} \mathrm{~F} \mathrm{NMR}\left(471 \mathrm{MHz}, \mathrm{CDCl}_{3}\right) \delta-63.21$ (s, $\mathrm{F}_{3} \mathrm{C}$ ); ${ }^{15} \mathrm{~N} \mathrm{NMR}$ (from $\mathrm{HMBC}, 600 \mathrm{MHz}, \mathrm{CDCl}_{3}$ ) $\delta-14.2$; high resolution (HR)MS (FTMS + probe (p)ESI) calcd. for $\mathrm{C}_{23} \mathrm{H}_{28} \mathrm{NOF}_{3} \mathrm{Na}[\mathrm{M}]^{+}:$414.2021; found: 414.2018.

3a, 12a-trans-(5E,9E)-3-[4-(Trifluoromethyl)phenyl]5,9,12,12-tetramethyl-3a $H, 4 H, 7 H, 8 H, 11 H, 12 H, 12 a H-$ cycloundeca[d][1,2]oxazole (7)

Celadon-white wax; yield: 50\%; ${ }^{1} \mathrm{H}$ NMR $(500 \mathrm{MHz}$, $\left.\mathrm{CDCl}_{3}\right) \delta 7.83(\mathrm{~d}, 2 \mathrm{H}, J 8.0 \mathrm{~Hz}, \mathrm{H} 2$ ', H6'), $7.68(\mathrm{~d}, 2 \mathrm{H}$, $J 8.0$ Hz, H3', H5'), 5.00 (dd, 1H, J 11.5, 4.0 Hz, H1), 4.92 (d, $1 \mathrm{H}, J 10.9 \mathrm{~Hz}, \mathrm{H} 8), 4.57$ (d, 1H, J $2.8 \mathrm{~Hz}, \mathrm{H} 5$ ), 3.29 (ddd, $1 \mathrm{H}, J 10.5,2.8,1.8 \mathrm{~Hz}, \mathrm{H} 4), 2.32$ (ddd, 1H, $J 22.5,11.5$,
$4.8 \mathrm{~Hz}, \mathrm{H} 11 \mathrm{a}), 2.24$ (dm, 1H, J 12.3 Hz, H10a), 2.23-2.17 (m, 1H, H3a), 2.13 (dd, 1H, J 10.5, 1.8 Hz, H3b), 2.11-2.07 (m, 2H, H7a, H11b), 1.98 (dd, 1H, J 12.3, 4.8 Hz, H10b), 1.96 (dd, 1H, J 12.0, $4.8 \mathrm{~Hz}, \mathrm{H} 7 \mathrm{~b}), 1.65$ (s, 3H, H16), 1.55 (s, 3H, H15), 1.22 (s, 3H, H13), 0.74 (s, 3H, H14); ${ }^{13} \mathrm{C}$ NMR $\left(125.9 \mathrm{MHz}, \mathrm{CDCl}_{3}\right.$ ) $\delta 158.0(\mathrm{C} 12), 133.7$ (C9), 133.0 (C2), 132.4 (q, $J 1.5 \mathrm{~Hz}, \mathrm{Cl}$ ') 131.4 (q, $J 32.7 \mathrm{~Hz}$, C4'), 127.7 (C1), 126.9 (C2', C6'), 125.9 (q, J3.4 Hz, C3', C5'), 123.5 (q, J 272.4 Hz, F $\mathrm{C}_{3}$ ), 123.0 (C8), 93.3 (C5), 46.0 (C4), 43.1 (C3), 38.9 (C10), 37.6 (C7), 25.9 (C11), 24.6 (C13), 22.7 (C14), 16.7 (C16), 16.5 (C15); ${ }^{19} \mathrm{~F}$ NMR $\left(471 \mathrm{MHz}, \mathrm{CDCl}_{3}\right) \delta-63.26\left(\mathrm{~s}, \mathrm{~F}_{3} \mathrm{C}\right.$ ); ${ }^{15} \mathrm{~N}$ NMR (from $\mathrm{HMBC}, 600 \mathrm{MHz}, \mathrm{CDCl}_{3}$ ) $\delta-9.57$; HRMS (FTMS + pESI) calcd. for: $\mathrm{C}_{23} \mathrm{H}_{28} \mathrm{NOF}_{3} \mathrm{H}[\mathrm{M}]^{+}: 392.2201$; found: 392.2197 .

3a,12a-trans-5,5,12a-Trimethyl-3,4'-bis[4-(trifluoromethyl) phenyl]-3a, 4, 5, 8, 10,11,12,12a-octahydro-3' $\mathrm{H}$ spiro[cycloundeca[d][1,2]oxazole-9,2'-[1,5]oxazole] (9)

Celadon-brown oil, yield: 55\%; ${ }^{1} \mathrm{H}$ NMR $(500 \mathrm{MHz}$, $\mathrm{CDCl}_{3}$ ) $\delta 7.80$ (d, 2H, J 8.3 Hz, H2", H6"), 7.69 (d, 2H, $J 8.0$ Hz, H3', H5'), 7.67 (d, 2H, J 8.3 Hz, H3", H5"), 7.65 (d, 2H, J 8.0 Hz, H2', H6'), 5.58 (d, 1H, J 15.5 Hz, H5), $5.43(\mathrm{ddd}, 1 \mathrm{H}, J 15.5,9.0,5.0 \mathrm{~Hz}, \mathrm{H} 4), 3.32(\mathrm{t}, 1 \mathrm{H}, J 5.5 \mathrm{~Hz}$, H8), 3.31 (d, 1H, J 16.5 Hz, H14a), 3.21 (d, 1H, J $16.5 \mathrm{~Hz}$, H14b), 2.66-2.63 (m, 2H, H3a, H3b), 2.02-1.97 (m, 1H, H1a), 1.91-1.85 (m, 2H, H10a, H11a), 1.80 (dd, 1H, J 12.5, $6.5 \mathrm{~Hz}, \mathrm{H} 10 \mathrm{~b}), 1.76$ (dd, 1H, J 15.5, 6.8 Hz, H7a), 1.67-1.58 (m, 2H, H1b, H11b), 1.46 (d, 1H, J 15.5 Hz, H7b), 1.41 (s, 3H, H17), 1.17 (s, 3H, H16), 1.02 (s, 3H, H15); ${ }^{13} \mathrm{C}$ NMR (125.9 MHz, $\mathrm{CDCl}_{3}$ ) $\delta 160.8$ (C12), 154.7 (C13), 141.7 (C5), 134.0 (C1'), 133.4 (C1'), 131.5 (q, J $32.5 \mathrm{~Hz}, \mathrm{C} 4$ '), 131.1 (q, J $32.1 \mathrm{~Hz}, \mathrm{C} 4$ ”), 128.0 (C2', C6'), 126.7 (C2”, C6”), 125.7 (q, J3.8 Hz, C3', C5'), 125.5 (q, J3.8 Hz, C3", C5”), 123.9 (q, J 272.1 Hz, F C-Ar'), 123.8 (q, J $272.1 \mathrm{~Hz}$, $\mathrm{F}_{3} \mathrm{C}-\mathrm{Ar}$ ”), 122.2 (C4), 91.4 (C9), 90.4 (C2), 47.5 (C8), 43.1 (C14), 42.8 (C3), 38.1 (C10), 38.05 (C1), 38.0 (C7), 37.3 (C6), 30.6 (C15), 24.8 (C16), 23.5 (C17), 16.9 (C11); MS $\left(\right.$ FTMS + pESI) $601\left([\mathrm{M}+\mathrm{Na}]^{+}, 35\right), 577\left([\mathrm{M}-\mathrm{H}]^{+}, 75\right)$; anal. calcd. for $\mathrm{C}_{31} \mathrm{H}_{32} \mathrm{~F}_{6} \mathrm{~N}_{2} \mathrm{O}_{2}[\mathrm{M}]^{+}: \mathrm{C}, 64.35 \% ; \mathrm{H}, 5.57 \%$; found: C, $64.19 \%$; H, $5.39 \%$.

(1S,5E,9R)-6,10,10-Trimethyl-4'-[4-(trifluoromethyl)phenyl]3'H-spiro[bicyclo[7.2.0]undecane-2,2'-[1,5]oxazole]-5-ene (11)

White wax; yield: $45 \%$; ${ }^{1} \mathrm{H}$ NMR $\left(500 \mathrm{MHz}, \mathrm{CDCl}_{3}\right.$ ) $\delta 7.83$ (d, 2H, J 8.2 Hz, H2', H6'), 7.67 (d, 2H, J 8.2 Hz, H3', H5'), 5.25-5.16 (m, 1H, H4), 3.32 (d, 1H, J $16.0 \mathrm{~Hz}$, H13a), 3.19 (d, 1H, J 16.0 Hz, H13b), 2.45-2.28 (m, 2H, H11, H3a), 2.14-2.08 (m, 1H, H6a), 2.09-2.03 (m, 1H, H2a), 2.01-1.96 (m, 1H, H3b), 1.99-1.93 (m, 2H, H2b, H6b), 1.81 (dd, 1H, J 12.0, $8.5 \mathrm{~Hz}, \mathrm{H} 10 \mathrm{a}$ ), 1.71 (s, 3H, 
$\mathrm{H} 14), 1.64-1.58$ (m, 2H, H8, H7a), 1.58-1.50 (m, 1H, H7b), 1.33-1.24 (m, 1H, H10b), 0.97 (s, 3H, H15), 0.92 (s, 3H, $\mathrm{H} 16) ;{ }^{13} \mathrm{C} \mathrm{NMR}\left(125.9 \mathrm{MHz}, \mathrm{CDCl}_{3}\right) \delta 154.1(\mathrm{C} 12), 137.8$ (C1'), 133.8 (C5), 131.1 (q, J 32.5 Hz, C4'), 126.6 (C2', C6'), 125.6 (q, J 3.9 Hz, C3', C5'), 123.9 (q, J 270.5 Hz, $\left.\mathrm{F}_{3} \mathrm{C}\right), 120.2(\mathrm{C} 4), 95.1$ (C1), 49.6 (C11), 48.5 (C8), 40.1 (C6), 38.7 (C2), 36.8 (C10), 36.0 (C13), 32.0 (C9), 30.5 (C15), 29.8 (C7), 23.5 (C16), 21.6 (C3), 16.0 (C14); HRMS (FTMS + pESI) calcd. for $\mathrm{C}_{23} \mathrm{H}_{28} \mathrm{NOF}_{3} \mathrm{Na}[\mathrm{M}]^{+}: 414.2021$; found: 414.2028 .

(1'S,4'R,7'S,11'R)-1',5',5'-Trimethyl-4-12'-bis[4-(trifluoromethyl)phenyl]-3H-14'-oxa-13'-azaspiro[1,5-oxazole-2,8tricyclo[9.3.0.0 $\left.0^{4,7}\right]$ tetradecan]-12'-ene (12)

Yellowish semisolid, yield: 30\%; ${ }^{1} \mathrm{H}$ NMR $(500 \mathrm{MHz}$, $\mathrm{CDCl}_{3}$ ) $\delta 7.80$ (d, 2H, J 8.3 Hz, H2", H6"), 7.71-7.70 (m, 2H, H3', H5'), 7.71 (d, 2H, J 8.3 Hz, H3", H5"), 7.70-7.69 (m, H2', H6'), 3.43 (d, 1H, J 16.0 Hz, H13a), 3.20 (d, 1H, $J 16.0 \mathrm{~Hz}, \mathrm{H} 13 \mathrm{~b}$ ), 3.25 (dm, 1H, J12.5 Hz, H4), 2.56 (app. q, $1 \mathrm{H}, J 9.3 \mathrm{~Hz}, \mathrm{H} 8), 2.23-2.17$ (m, 1H, H6a), 2.17-2.09 (m, 2H, H2a, H6b), 1.97-1.89 (m, 1H, H11), 1.86-1.77 (m, 3H, H2b, H7a, H10a), 1.76-1.68 (m, 1H, H7b), 1.49 (s, 3H, H15), 1.33-1.26 (m, 3H, H3a, H3b, H10b), 1.03 (s, 3H, H16), 0.99 (s, 3H, H17); ${ }^{13} \mathrm{C}$ NMR (125.9 MHz, $\left.\mathrm{CDCl}_{3}\right) \delta 158.9$ (C14), 153.8 (C12), 133.7 (C1'), 133.2 (q, J 1.1 Hz, C1"), 131.6 (q, J 32.6 Hz, C4'), 131.5 (q, J 32.6 Hz, C4"), 127.4 (C2', C6'), 126.5 (C2", C6”), 125.7 (q, J3.7 Hz, C3', C5'), 125.7 (q, J3.7 Hz, C3", C5”), 123.8 (q, J $272.4 \mathrm{~Hz}, \mathrm{~F}_{3} \mathrm{C}-\mathrm{Ar}$ '), 123.7 (q, J $272.4 \mathrm{~Hz}, \mathrm{~F}_{3} \mathrm{C}-\mathrm{Ar}^{\prime \prime}$ ), 92.9 (C1), 90.2 (C5), 50.0 (C4), 48.7 (C11), 46.8 (C8), 36.6 (C2), 36.5 (C6), 35.9 (C13), 35.1 (C10), 33.9 (C9), 29.9 (C16), 29.6 (C3), 22.3 (C7), 22.2 (C17), 19.6 (C15); MS (FTMS + pESI) $601\left([\mathrm{M}+\mathrm{Na}]^{+}, 20\right), 577\left([\mathrm{M}-\mathrm{H}]^{+}\right.$, 12); anal. calcd. for $\mathrm{C}_{31} \mathrm{H}_{32} \mathrm{~F}_{6} \mathrm{~N}_{2} \mathrm{O}_{2}$ : C, 64.35\%; $\mathrm{H}, 5.57 \%$; found: C, $64.58 \%$; $\mathrm{H}, 5.42 \%$.

(6E,10E)-3a,13a-cis-3-[4-(Trifluoromethyl)phenyl]$3 a H, 4 H, 5 H, 8 H, 9 H, 12 H, 13 H, 13 a H$-cyclododeca[d][1,2] oxazole $(14,15)$

White-celadon dense oil (ratio of $\mathbf{1 4}$ and $\mathbf{1 5}$ isomers: 53:47), yield: $62 \%$; ${ }^{1} \mathrm{H}$ and ${ }^{13} \mathrm{C}$ NMR see Tables 1 and 2; MS (FTMS + pESI) $372\left([\mathrm{M}+\mathrm{Na}]^{+}, 30\right), 350\left([\mathrm{M}+\mathrm{H}]^{+}\right.$, 80), $227\left(\left[\mathrm{M}-\mathrm{C}_{6} \mathrm{H}_{4} \mathrm{CF}_{3}\right]^{+}\right.$, 99); anal. calcd. for $\mathrm{C}_{20} \mathrm{H}_{22} \mathrm{~F}_{3} \mathrm{NO}$ : C, $68.75 \%$; H, $6.35 \%$; found: C, $68.93 \%$; H, $6.49 \%$.

(6E,10E)-3a,13a-trans-3-[4-(Trifluoromethyl)phenyl]$3 a, 4,5,8,9,12,13,13 a-o c t a h y d r o c y c l o d o d e c a[d][1,2]$ oxazole $(16,17)$

White wax (ratio of $\mathbf{1 6}$ and $\mathbf{1 7}$ isomers: 60:40), yield: $38 \%$; ${ }^{1} \mathrm{H}$ and ${ }^{13} \mathrm{C}$ NMR see Tables 1 and 2; anal. calcd. for

Table 1. ${ }^{1} \mathrm{H}$ NMR signals of $\mathbf{1 4}, \mathbf{1 5}, \mathbf{1 6}$, and $17\left(500 \mathrm{MHz}, \mathrm{CDCl}_{3}\right)$

\begin{tabular}{|c|c|c|c|c|}
\hline \multirow{2}{*}{$\mathrm{H}$} & \multicolumn{4}{|c|}{$\delta / \mathrm{ppm}(\mathrm{J} / \mathrm{Hz})$} \\
\hline & 14 & 15 & 16 & 17 \\
\hline 1 & $5.09-4.98(\mathrm{~m})$ & $5.53-5.45(\mathrm{~m})$ & $3.37(\mathrm{ddd}, 10.5,3.0,1.5)$ & $4.61(\mathrm{ddd}, 10.5,4.5,2.0)$ \\
\hline 2 & $5.43-5.30(\mathrm{~m})$ & $5.41-5.27(\mathrm{~m})$ & $4.66(\mathrm{dm}, 10.5)$ & $3.46(\mathrm{dm}, 10.5)$ \\
\hline $3 a$ & $2.14-2.07(\mathrm{~m})$ & $2.12-2.05(\mathrm{~m})$ & $1.84-1.79(\mathrm{~m})$ & $1.65-1.60(\mathrm{~m})$ \\
\hline $3 b$ & $2.05-1.99(\mathrm{~m})$ & $2.02-1.96(\mathrm{~m})$ & $1.68-1.62(\mathrm{~m})$ & $1.57-1.51(\mathrm{~m})$ \\
\hline $4 a$ & $2.12-2.08(\mathrm{~m})$ & $2.12-2.08(\mathrm{~m})$ & $2.16-2.12(\mathrm{~m})$ & $2.28-2.21(\mathrm{~m})$ \\
\hline $4 \mathrm{~b}$ & $2.20-2.14(\mathrm{~m})$ & $2.02-1.96(\mathrm{~m})$ & $2.05-1.98(\mathrm{~m})$ & $2.20-2.18(\mathrm{~m})$ \\
\hline 5 & $5.23-5.12(\mathrm{~m})$ & $5.38-5.30(\mathrm{~m})$ & $5.52-5.42(\mathrm{~m})$ & $5.30-5.22(\mathrm{~m})$ \\
\hline 6 & $5.43-5.30(\mathrm{~m})$ & $5.23-5.12(\mathrm{~m})$ & $5.40-5.35(\mathrm{~m})$ & $5.52-5.42(\mathrm{~m})$ \\
\hline $7 \mathrm{a}$ & $2.06-2.00(\mathrm{~m})$ & $2.22-2.14(\mathrm{~m})$ & $2.37-2.32(\mathrm{~m})$ & $2.27-2.20(\mathrm{~m})$ \\
\hline $7 b$ & $2.02-1.95(\mathrm{~m})$ & $2.11-2.05(\mathrm{~m})$ & $2.15-2.10(\mathrm{~m})$ & $2.16-2.10(\mathrm{~m})$ \\
\hline $8 \mathrm{a}$ & $2.24-2.18(\mathrm{~m})$ & $1.31-1.22(\mathrm{~m})$ & $2.29-2.20(\mathrm{~m})$ & $2.15-2.10(\mathrm{~m})$ \\
\hline $8 b$ & $1.84-1.76(\mathrm{~m})$ & $1.05-1.00(\mathrm{~m})$ & $2.15-2.10(\mathrm{~m})$ & $2.12-2.06(\mathrm{~m})$ \\
\hline 9 & $4.24(\mathrm{ddd}, 12.5,10.1,1.8)$ & $3.41(\mathrm{dm}, 11.2)$ & $5.53-5.42(\mathrm{~m})$ & $5.53-5.43(\mathrm{~m})$ \\
\hline 10 & $3.20(\mathrm{dd}, 12.5,3.2)$ & $4.35-4.30(\mathrm{~m})$ & $5.29-5.22(\mathrm{~m})$ & $5.40-5.34(\mathrm{~m})$ \\
\hline $11 \mathrm{a}$ & $1.48-1.36(\mathrm{~m})$ & $2.22-2.14(\mathrm{~m})$ & $2.28-2.21(\mathrm{~m})$ & $2.41-2.30(\mathrm{~m})$ \\
\hline $11 \mathrm{~b}$ & $1.07-0.98(\mathrm{~m})$ & $1.82-1.77(\mathrm{~m})$ & $2.15-2.10(\mathrm{~m})$ & $2.15-2.10(\mathrm{~m})$ \\
\hline $12 \mathrm{a}$ & $2.24-2.18(\mathrm{~m})$ & $2.23-2.16(\mathrm{~m})$ & $1.67-1.62(\mathrm{~m})$ & $1.87-1.83(\mathrm{~m})$ \\
\hline $12 b$ & $2.02-1.95(\mathrm{~m})$ & $2.02-1.95(\mathrm{~m})$ & $1.59-1.53(\mathrm{~m})$ & $1.74-1.68(\mathrm{~m})$ \\
\hline $2^{\prime}, 6$ & $7.80(\mathrm{~d}, 8.1)$ & $8.09(\mathrm{dm}, 8.1)$ & $7.83(\mathrm{~d}, 8.0)$ & $7.79(\mathrm{~d}, 8.0)$ \\
\hline 3,5, & $7.67(\mathrm{dd}, 8.1,2.6)$ & $7.71(\mathrm{dm}, 8.1)$ & $7.68(\mathrm{~d}, 8.0)$ & $7.68(\mathrm{~d}, 8.0)$ \\
\hline
\end{tabular}

$\delta$ : chemical shift; $J$ : coupling constant. 
Table 2. ${ }^{13} \mathrm{C}$ NMR signals of $\mathbf{1 4}, \mathbf{1 5}, \mathbf{1 6}$, and $\mathbf{1 7}\left(125.8 \mathrm{MHz}, \mathrm{CDCl}_{3}\right)$

\begin{tabular}{|c|c|c|c|c|}
\hline \multirow{2}{*}{$\mathrm{C}$} & \multicolumn{4}{|c|}{$\delta / \operatorname{ppm}(J / \mathrm{Hz})$} \\
\hline & 14 & 15 & 16 & 17 \\
\hline 1 & 130.5 & 130.9 & 50.4 & 85.9 \\
\hline 2 & 131.8 & 129.3 & 86.0 & 49.7 \\
\hline 3 & 32.3 & 31.6 & 31.4 & 28.4 \\
\hline 4 & 31.5 & 31.7 & 31.8 & 30.2 \\
\hline 5 & 129.3 & 131.5 & 132.2 & 129.1 \\
\hline 6 & 130.9 & 129.0 & 129.7 & 130.0 \\
\hline 7 & 27.1 & 31.2 & 24.9 & 32.0 \\
\hline 8 & 29.7 & 28.6 & 22.4 & 24.9 \\
\hline 9 & 87.2 & 53.9 & 131.3 & 130.9 \\
\hline 10 & 56.9 & 87.4 & 129.2 & 129.7 \\
\hline 11 & 30.6 & 29.7 & 22.4 & 24.9 \\
\hline 12 & 31.1 & 27.0 & 28.4 & 32.5 \\
\hline 13 & 159.2 & 159.4 & 159.5 & 159.7 \\
\hline 1 ' & $132.5(\mathrm{q}, 2.4)$ & $132.8(\mathrm{q}, 2.4)$ & $132.7(\mathrm{q}, 1.3)$ & $132.8(\mathrm{q}, 1.3)$ \\
\hline $2^{\prime}, 6^{\prime}$ & 127.3 & 127.1 & 127.2 & 127.2 \\
\hline $3,5^{\prime}$ & $125.8(\mathrm{q}, 3.8)$ & $125.7(\mathrm{~m})$ & $125.8(\mathrm{q}, 3.7)$ & $125.8(\mathrm{q}, 3.7)$ \\
\hline 4' & $131.2(\mathrm{q}, 32.7)$ & $131.1(\mathrm{q}, 32.6)$ & $131.5(\mathrm{~m})$ & $131.4(\mathrm{~m})$ \\
\hline $\mathrm{CF}_{3}$ & $123.7(\mathrm{q}, 270.9)$ & $124.1(\mathrm{q}, 272.0)$ & $123.7(q, 270.6)$ & $123.7(\mathrm{q}, 270.6)$ \\
\hline
\end{tabular}

$\delta$ : chemical shift; $J$ : coupling constant.

$\mathrm{C}_{20} \mathrm{H}_{22} \mathrm{~F}_{3} \mathrm{NO}: \mathrm{C}, 68.75 \%$; H, 6.35\%; found: $\mathrm{C}, 68.65 \%$; H, $6.47 \%$.

3a, 9 a - cis-3-[ 4 - (Trifluoromethyl)phenyl] $3 a H, 4 H, 5 H, 6 H, 7 H, 9 a H$-cycloocta[d][1,2]oxazole (19); 3-[4-(trifluoromethyl)phenyl]-3a $\mathrm{H}, 6 \mathrm{H}, 7 \mathrm{H}, 8 \mathrm{H}, 9 \mathrm{H}, 9 \mathrm{aH}$ cycloocta[d][1,2]oxazole (20)

Celadon-yellow oil (ratio of $\mathbf{1 9 / 2 0}$ isomers 5/1), yield: 45\%; 19: ${ }^{1} \mathrm{H}$ NMR $\left(600 \mathrm{MHz}, \mathrm{CDCl}_{3}\right) \delta 7.71(\mathrm{~d}, 2 \mathrm{H}$, $J 8.3$ Hz, H3', H5'), 7.66 (d, 2H, J 8.3 Hz, H2', H6'), 5.87$5.82(\mathrm{~m}, 1 \mathrm{H}, \mathrm{H} 1), 5.60(\mathrm{dm}, J 11.8 \mathrm{~Hz}, 1 \mathrm{H}, \mathrm{H} 3), 5.55(\mathrm{dd}$, $1 \mathrm{H}, J 11.8,3.9 \mathrm{~Hz}, \mathrm{H} 2), 3.74$ (td, 1H, $J 10.1,1.5 \mathrm{~Hz}, \mathrm{H} 8$ ), 2.25-2.13 (m, 2H, H4), 1.90-1.80 (m, 2H, H7), 1.58-150 (m, 2H, H5); ${ }^{13} \mathrm{C}$ NMR (from HMBC, $150.8 \mathrm{MHz}, \mathrm{CDCl}_{3}$ )

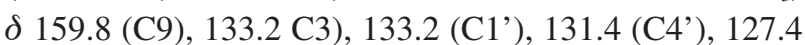
(C-2', C-6'), 126.7 (C2), 125.7 (C3', C5'), 83.2 (C1), 53.0 (C8), 30.2 (C7), 26.6 (C5), 25.8 (C4), 24.6 (C16); ${ }^{15} \mathrm{~N}$ NMR (from HMBC, $600 \mathrm{MHz}, \mathrm{CDCl}_{3}$ ) $\delta-14.10(\mathrm{~s}, 1 \mathrm{~N},=\mathrm{N}-\mathrm{O}$ ); 20: ${ }^{1} \mathrm{H}$ NMR $\left(600 \mathrm{MHz}, \mathrm{CDCl}_{3}\right) \delta 7.83(\mathrm{~d}, 2 \mathrm{H}, J 8.0 \mathrm{~Hz}$, H2', H6'), 7.64 (d, 2H, J 8.0 Hz, H3', H5'), 6.02-5.97 (m, $1 \mathrm{H}, \mathrm{H} 2$ ), 5.16 (ddd, 1H, J 11.1, 7.0, 0.8 Hz, H3), 4.61 (ddd, $1 \mathrm{H}, J 11.1,9.4,3.3 \mathrm{~Hz}, \mathrm{H} 8), 4.41$ (tm, 1H, J 8.0 Hz, H1), 2.28-2.20 (m, 2H, H4), 2.00-1.80 (m, H6, H7), 1.58-1.48 (m, $1 \mathrm{H}, \mathrm{H} 5)$; anal. calcd. for $\mathrm{C}_{16} \mathrm{H}_{16} \mathrm{~F}_{3} \mathrm{NO}$ : $\mathrm{C}, 65.08 \%$; $\mathrm{H}$, $5.46 \%$; found: C, $65.36 \%$; $\mathrm{H}, 5.67 \%$.
9-Ethenyl-5-[4-(trifluoromethyl)phenyl]-3-oxa-4azatricyclo[5.2.1.0 2,6]dec-4-ene (22)

White wax; yield: $12 \%$; ${ }^{1} \mathrm{H}$ NMR $\left(500 \mathrm{MHz}, \mathrm{CDCl}_{3}\right)$ $\delta 7.76$ (d, 2H, J 8.5 Hz, H2', H6'), 7.64 (d, 2H, J 8.5 Hz, H3', H5'), 5.94 (ddd, 1H, J 17.0, 10.5, 1.5 Hz, H2), 5.21 (ddd, $1 \mathrm{H}, J 10.5,1.5 \mathrm{~Hz}, \mathrm{H} 1 \mathrm{a}), 5.19$ (dd, $1 \mathrm{H}, J 17.0$, $1.5 \mathrm{~Hz}, \mathrm{H} 1 \mathrm{~b}), 4.65$ (dm, 1H, J $8.3 \mathrm{~Hz}, \mathrm{H} 7$ ), 3.78 (dm, $1 \mathrm{H}, J 8.3 \mathrm{~Hz}, \mathrm{H} 6), 2.65$ (d, 1H, J 5.5 Hz, H8), 2.67-2.61 (m, 1H, H3), 2.45 (d, 1H, J $3.0 \mathrm{~Hz}, \mathrm{H} 5), 1.85$ (ddd, 1H, $J$ 13.3, 11.0, $5.5 \mathrm{~Hz}, \mathrm{H} 4 \mathrm{a}), 1.57$ (dm, 1H, J 9.3 Hz, H9a), 1.37 (dm, 1H, $J 9.3 \mathrm{~Hz}, \mathrm{H} 9 \mathrm{~b}$ ), 1.03 (ddd, $1 \mathrm{H}, J 13.3,5.5$, $2.0 \mathrm{~Hz}, \mathrm{H} 4 \mathrm{~b}) ;{ }^{13} \mathrm{C}$ NMR $\left(125.9 \mathrm{MHz}, \mathrm{CDCl}_{3}\right) \delta 156.4$ (C10), 139.1 (C2), 132.8 (q, J 1.3 Hz, C1'), 131.3 (q, $J 32.7$ Hz, C4'), 126.9 (C2', C6'), 125.7 (q, J3.8 Hz, C3', C5'), 123.8 (q, J 272.3 Hz, $\mathrm{F}_{3} \mathrm{C}$ ), 116.3 (C1), 88.4 (C7), 50.6 (C6), 44.6 (C5), 44.0 (C8), 41.9 (C3), 33.5 (C9), 27.8 (C4); anal. calcd. for $\mathrm{C}_{17} \mathrm{H}_{16} \mathrm{~F}_{3} \mathrm{NO}$ : C, 66.44\%; $\mathrm{H}, 5.25 \%$; found: $\mathrm{C}, 66.61 \%$; $\mathrm{H}, 5.17 \%$.

8-Ethenyl-5-[4-(trifluoromethyl)phenyl]-3-oxa-4azatricyclo[5.2.1.0 $\left.0^{2,6}\right]$ dec-4-ene (23)

White-yelowish wax; yield: $30 \%$; ${ }^{1} \mathrm{H}$ NMR $(500 \mathrm{MHz}$, $\left.\mathrm{CDCl}_{3}\right) \delta 7.83\left(\mathrm{~d}, 2 \mathrm{H}, J 8.0 \mathrm{~Hz}, \mathrm{H} 2^{\prime}, \mathrm{H}^{\prime}\right), 7.65(\mathrm{~d}, 2 \mathrm{H}$, $J 8.0 \mathrm{~Hz}, \mathrm{H} 3$ ', H5'), 5.85 (ddd, $1 \mathrm{H}, J 17.3,5.5,1.5 \mathrm{~Hz}$, H2), 5.13 (dd, 1H, J 17.3, $1.5 \mathrm{~Hz}, \mathrm{H} 1 \mathrm{a}), 5.12$ (d, 1H, J 10.5, 
$1.5 \mathrm{~Hz}, \mathrm{H} 1 \mathrm{~b}), 4.92$ (d, 1H, J $8.5 \mathrm{~Hz}, \mathrm{H} 6), 3.51$ (d, 1H, $J ~ 8.5 \mathrm{~Hz}, \mathrm{H} 7$ ), 2.70 (d, 1H, J 4.8 Hz, H5), 2.69-2.63 (m, 1H, H3), 2.51 (d, 1H, J 4.5 Hz, H8), 1.91 (ddd, 1H, J 15.4, 11.0, $4.8 \mathrm{~Hz}, \mathrm{H} 4 \mathrm{a}$ ), 1.56 (app. dq, $1 \mathrm{H}, J 10.5,2.0 \mathrm{~Hz}, \mathrm{H} 9 \mathrm{a}$ ), 1.35 (dt, 1H, $J$ 10.5, $2.0 \mathrm{~Hz}, \mathrm{H} 9 \mathrm{~b}$ ), 1.30 (ddd, 1H, $J 15.4$, $4.8,2.5 \mathrm{~Hz}, \mathrm{H} 4 \mathrm{~b}) ;{ }^{13} \mathrm{C}$ NMR $\left(125.9 \mathrm{MHz}, \mathrm{CDCl}_{3}\right) \delta 156.0$ (C10), 138.8 (C2), 132.8 (C1'), 131.2 (q, J 32.7 Hz, C4'), 127.0 (C2', C6'), 125.7 (q, J 3.9 Hz, C3', C5'), 123.9 (q, $J 272.2 \mathrm{~Hz}, \mathrm{~F}_{3} \mathrm{C}$ ), 115.5 (C1), 85.0 (C6), 56.8 (C7), 48.2 (C8), 40.3 (C5), 40.0 (C3), 33.6 (C9), 32.4 (C4); anal. calcd. for $\mathrm{C}_{17} \mathrm{H}_{16} \mathrm{~F}_{3} \mathrm{NO}$ : $\mathrm{C}, 66.44 \%$; $\mathrm{H}, 5.25 \%$; found: $\mathrm{C}$, $66.30 \% ; \mathrm{H}, 5.39 \%$.

5-[4-(Trifluoromethyl)phenyl]-8-endo-\{3-[4-(trifluoromethyl) phenyl]-4,5-dihydro-1,2-oxazol-5-yl\}-3-oxa-4azatricyclo[5.2.1.0 2,6]dec-4-ene (24)

Celadon wax; yield: $11 \%$; ${ }^{1} \mathrm{H}$ and ${ }^{13} \mathrm{C}$ NMR see Tables 3 and 4; anal. calcd. for $\mathrm{C}_{25} \mathrm{H}_{20} \mathrm{~F}_{6} \mathrm{~N}_{2} \mathrm{O}_{2}: \mathrm{C}, 60.73 \% ; \mathrm{H}, 4.87 \%$; found: C, $60.92 \%$; H, $4.91 \%$.

5-[4-(Trifluoromethyl)phenyl]-9-endo-\{3-[4-(trifluoromethyl) phenyl]-4,5-dihydro-1,2-oxazol-5-yl\}-3-oxa-4azatricyclo[5.2.1.0 $\left.0^{2,6}\right]$ dec-4-ene (25)

Celadon wax; yield: 7\%; ${ }^{1} \mathrm{H}$ and ${ }^{13} \mathrm{C}$ NMR see Tables 3 and 4; anal. calcd. for $\mathrm{C}_{25} \mathrm{H}_{20} \mathrm{~F}_{6} \mathrm{~N}_{2} \mathrm{O}_{2}: \mathrm{C}, 60.73 \% ; \mathrm{H}, 4.87 \%$; found: $\mathrm{C}, 60.89 \% ; \mathrm{H}, 5.03 \%$.
5-[4-(Trifluoromethyl)phenyl]-9-exo-\{3-[4-(trifluoromethyl) phenyl]-4,5-dihydro-1,2-oxazol-5-yl\}-3-oxa-4azatricyclo[5.2.1.0 $\left.0^{2,6}\right] \mathrm{dec}-4$-ene (26)

Celadon wax; yield: $8 \%$; ${ }^{1} \mathrm{H}$ and ${ }^{13} \mathrm{C}$ NMR see Tables 3 and 4; anal. calcd. for $\mathrm{C}_{25} \mathrm{H}_{20} \mathrm{~F}_{6} \mathrm{~N}_{2} \mathrm{O}_{2}: \mathrm{C}, 60.73 \% ; \mathrm{H}, 4.87 \%$; found: $\mathrm{C}, 60.54 \%$; $\mathrm{H}, 5.02 \%$.

5-[4-(Trifluoromethyl)phenyl]-8-exo-\{3-[4-(trifluoromethyl) phenyl]-4,5-dihydro-1,2-oxazol-5-yl\}-3-oxa-4azatricyclo[5.2.1.0 $\left.0^{2,6}\right]$ dec-4-ene (27)

Celadon wax; yield: $12 \%$; ${ }^{1} \mathrm{H}$ and ${ }^{13} \mathrm{C}$ NMR see Tables 3 and 4; anal. calcd. for $\mathrm{C}_{25} \mathrm{H}_{20} \mathrm{~F}_{6} \mathrm{~N}_{2} \mathrm{O}_{2}: \mathrm{C}, 60.73 \% ; \mathrm{H}, 4.87 \%$; found: $\mathrm{C}, 60.63 \%$; $\mathrm{H}, 4.75 \%$.

\section{Fungicidal testing}

The compounds were screened for fungicidal activity in vitro. The tests were carried out for Fusarium culmorum (F. c.), Phytophthora cactorum (P. c.), Rhizoctonia solani (Rh. s.), and Botrytis cinerea (B. c.), and involved determination of mycelial growth retardation in potato glucose agar (PGA). Stock solutions of test chemicals in acetone were added to agar medium to give a concentration of $200 \mathrm{mg} \mathrm{L}^{-1}$ and dispersed into Petri dishes. Four discs containing the test fungus were placed at intervals on the surface of the solidified agar and the dishes were then

Table 3. ${ }^{1} \mathrm{H}$ NMR signals of $\mathbf{2 4 , 2 5 ,} 26$ and $27\left(500 \mathrm{MHz}, \mathrm{CDCl}_{3}\right)$

\begin{tabular}{|c|c|c|c|c|}
\hline \multirow{2}{*}{$\mathrm{H}$} & \multicolumn{4}{|c|}{$\delta / \operatorname{ppm}(J / \mathrm{Hz})$} \\
\hline & 24 & 25 & 26 & 27 \\
\hline $1 \mathrm{a}$ & $3.56(\mathrm{dd}, 16.3,10.5)$ & $3.54(\mathrm{dd}, 16.5,10.5)$ & $3.53(\mathrm{dd}, 16.5,10.3)$ & $3.53(\mathrm{dd}, 16.3,10.3)$ \\
\hline $1 b$ & $3.08(\mathrm{dd}, 16.3,9.4)$ & $3.08(\mathrm{dd}, 16.5,9.0)$ & $3.07(\mathrm{dd}, 16.5,8.0)$ & $3.07(\mathrm{dd}, 16.3,8.3)$ \\
\hline 2 & $5.01(\mathrm{ddd}, 10.5,9.4,4.8)$ & $4.86(\mathrm{ddd}, 10.5,9.0,6.5)$ & $4.73-4.65(\mathrm{~m})$ & 4.73-4.65 (m) \\
\hline 3 & $2.29-2.22(\mathrm{~m})$ & $2.30-2.24(\mathrm{~m})$ & $2.25-2.19(\mathrm{~m})$ & $2.28-2.19(\mathrm{~m})$ \\
\hline $4 a$ & $1.83(\mathrm{ddd}, 12.0,12.0 ; 5.5)$ & 1.92(ddd, $12.0,8.0,4.0)$ & $1.90(\mathrm{ddd}, 10.0,7.5,5.4)$ & $1.90(\mathrm{td}, 12.0,5.0)$ \\
\hline $4 b$ & $1.18(\mathrm{ddd}, 12.0,5.8,2.5)$ & $1.40(\mathrm{dm}, 12.0)$ & $0.79(\mathrm{ddd}, 10.0,5.4,3.5)$ & $0.79(\mathrm{dm}, 12.0)$ \\
\hline 5 & $2.71(\mathrm{~d}, 5.0)$ & $2.77(\mathrm{~d}, 4.0)$ & $2.82(\mathrm{bd}, 3.5)$ & $2.82(\mathrm{~d}, 2.5)$ \\
\hline 6 & $4.80(\mathrm{~d}, 8.3)$ & $5.16(\mathrm{~d}, 8.3)$ & $3.99(\mathrm{~d}, 8.3)$ & $3.99(\mathrm{~d}, 8.3)$ \\
\hline 7 & $4.20(\mathrm{~d}, 8.3)$ & $3.69(\mathrm{~d}, 8.3)$ & $4.70(\mathrm{~d}, 8.3)$ & $4.70(\mathrm{~d}, 8.3)$ \\
\hline 8 & $2.54(\mathrm{~d}, 3.5)$ & $2.56(\mathrm{bs})$ & $2.70(\mathrm{~d}, 5.0)$ & $2.70(\mathrm{~d}, 5.0)$ \\
\hline $9 \mathrm{a}$ & $1.60(\mathrm{dm}, 11.0)$ & $1.65-1.62(\mathrm{~m})$ & $1.62(\mathrm{dm}, 11.0)$ & $1.62(\mathrm{dm}, 11.0)$ \\
\hline $9 b$ & $1.36(\mathrm{dm}, 11.0)$ & $1.37-1.32(\mathrm{~m})$ & $1.33(\mathrm{dm}, 11.0)$ & $1.33(\mathrm{dm}, 11.0)$ \\
\hline $2^{\prime}, 6^{\prime}$ & $7.80(\mathrm{~d}, 8.5)$ & $7.83(\mathrm{~d}, 8.5)$ & $7.94(\mathrm{~d}, 8.5)$ & $7.93(\mathrm{~d}, 8.0)$ \\
\hline $3^{\prime}, 5^{\prime}$ & $7.69(\mathrm{~d}, 8.5)$ & $7.66(\mathrm{~d}, 8.5)$ & $7.68(\mathrm{~d}, 8.5)$ & $7.69(\mathrm{~d}, 8.0)$ \\
\hline $2 ", 6 "$ & $7.79(\mathrm{~d}, 8.0)$ & $7.78(\mathrm{~d}, 8.3)$ & $7.80(\mathrm{~d}, 8.0)$ & $7.80(\mathrm{~d}, 8.0)$ \\
\hline $3 ", 5 "$ & $7.68(\mathrm{~d}, 8.0)$ & $7.68(\mathrm{~d}, 8.3)$ & $7.69(\mathrm{~d}, 8.0)$ & $7.67(\mathrm{~d}, 8.0)$ \\
\hline
\end{tabular}

$\delta$ : chemical shift; $J$ : coupling constant. 
Table 4. ${ }^{13} \mathrm{C}$ NMR signals of $\mathbf{2 4 ,} \mathbf{2 5}, 26$ and $27\left(125.8 \mathrm{MHz}, \mathrm{CDCl}_{3}\right)$

\begin{tabular}{|c|c|c|c|c|}
\hline \multirow{2}{*}{$\mathrm{C}$} & \multicolumn{4}{|c|}{$\delta / \operatorname{ppm}(J / \mathrm{Hz})$} \\
\hline & 24 & 25 & 26 & 27 \\
\hline 1 & 40.1 & 39.9 & 39.7 & 39.7 \\
\hline 2 & 83.0 & 82.9 & 82.9 & 82.9 \\
\hline 3 & 43.9 & 42.2 & 43.9 & 43.9 \\
\hline 4 & 24.6 & 29.7 & 26.6 & 26.6 \\
\hline 5 & 44.0 & 46.7 & 41.4 & 41.4 \\
\hline 6 & 88.3 & 56.9 & 50.7 & 50.7 \\
\hline 7 & 51.2 & 84.8 & 87.9 & 87.9 \\
\hline 8 & 42.8 & 40.3 & 43.5 & 43.5 \\
\hline 9 & 33.9 & 33.8 & 33.2 & 33.2 \\
\hline 10 & 155.9 & 156.1 & 156.2 & 156.2 \\
\hline 11 & 155.2 & 155.4 & 155.7 & 155.7 \\
\hline $1^{\prime}$ & 132.8 & 132.8 & 132.8 & 132.8 \\
\hline $2^{\prime}, 6^{\prime}$ & 126.9 & 127.1 & 127.2 & 127.9 \\
\hline $3^{\prime}, 5^{\prime}$ & $125.8(\mathrm{q}, 3.8)$ & $125.8(\mathrm{q}, 3.8)$ & $125.8(\mathrm{q}, 3.8)$ & $125.9(\mathrm{q}, 3.8)$ \\
\hline $4^{\prime}$ & $132.0(\mathrm{q}, 32.8)$ & $131.9(\mathrm{q}, 32.8)$ & $132.1(\mathrm{q}, 32.7)$ & $131.5(\mathrm{q}, 32.6)$ \\
\hline $\mathrm{CF}_{3}$, & $123.8(\mathrm{q}, 272.4)$ & $123.8(\mathrm{q}, 272.1)$ & $124.9(\mathrm{q}, 272.2)$ & $123.9(\mathrm{q}, 272.1)$ \\
\hline $1 "$ & 132.7 & 132.7 & 132.3 & 132.3 \\
\hline $2 ", 6 "$ & 126.9 & 126.9 & 126.9 & 126.9 \\
\hline $3 ", 5 "$ & $125.8(\mathrm{q}, 3.8)$ & $125.7(\mathrm{q}, 3.7)$ & $125.8(\mathrm{q}, 3.8)$ & $125.8(\mathrm{q}, 3.7)$ \\
\hline $\mathrm{CF}_{3}$, & $123.8(\mathrm{q}, 272.4)$ & $123.8(\mathrm{q}, 272.0)$ & $124.8(\mathrm{q}, 272.4)$ & $123.8(\mathrm{q}, 272.5)$ \\
\hline 4" & $131.5(\mathrm{q}, 32.7)$ & $131.5(\mathrm{q}, 35.7)$ & $131.3(\mathrm{q}, 32.6)$ & $132.0(\mathrm{q}, 32.7)$ \\
\hline
\end{tabular}

$\delta$ : chemical shift; $J$ : coupling constant.

inoculated for 4-8 days depending on the growth rate of the control samples, after which fungal growth was compared with that in untreated control samples. The fungicidal activity was expressed as the percentage of fungi linear growth inhibition compared to that of the control.

\section{Results and Discussion}

Structural analysis of the cycloadducts

We have examined [2 +3$]$ cycloaddition of 4-(trifluoromethyl)benzonitrile oxide (4) to polyunsaturated medium rings including 11-membered sesquiterpenes $\mathbf{5 , 8}$, and 10, 1,5,9-cyclododecatriene (13), 1,3-cyclooctadiene (18) and 5-vinyl-2-norbornene (21). The compounds described in this work are presented in Schemes 1-6 and Figure $1 .{ }^{1} \mathrm{H}$ and ${ }^{13} \mathrm{C}$ NMR chemical shifts and multiplicities of adducts 14-17 are shown, respectively, in Tables 1 and 2. ${ }^{1} \mathrm{H}$ and ${ }^{13} \mathrm{C}$ NMR chemical shifts of adducts 24-27 are shown, respectively, in Tables 3 and 4. The spectroscopic data for the other products are presented in Experimental.

Cycloaddition of the sesquiterpene $\mathrm{C}_{15}$ trans,trans,trans2,6,6,9-tetramethyl-1,4,8-cycloundecatriene (5) afforded two mono-adducts to different double bonds, 6 and 7 , in $3: 1$ ratio (Scheme 1, Figure 1). Structure 6 was assigned to the major isomer based on analysis of 2D NMR HSQC and COSY spectra. Presence of three $\mathrm{H}-\mathrm{C}$ correlations in an olefinic area in HSQC spectrum and presence of $\mathrm{HC}=\mathrm{HC}$ group in the COSY spectrum indicated that an addition to one of two tri-substituted double bonds took place. Observation of a $\mathrm{CH}^{1}-\mathrm{CH}_{2}{ }^{11}-\mathrm{CH}_{2}{ }^{10}$ and not of a $\mathrm{CH}^{8}-\mathrm{CH}_{2}{ }^{7}$ moiety in $\mathrm{COSY}$ spectrum showed that the addition occurred to $\mathrm{C} 1-\mathrm{C} 2$ double bond. Finally, regiochemistry of the cycloaddition was established as that shown in structure 6 by lack of $\mathrm{H}-\mathrm{C}$ correlation in HSQC spectrum for carbon 2 connected to isoxazoline oxygen atom. This direction of addition was favored by the orbital factors. The reaction is LUMO-dipole controlled and oxygen atom of the dipole tends to attack the more substituted carbon atom of the dipolarophile.

Structure 7 was assigned to the minor isomer based on analysis of 2D NMR HSQC, COSY and HMBC spectra. Presence of two H-C correlations in an olefinic area in HSQC spectrum proved the addition to the di-substituted C4-C5 double bond. Correlations in the COSY spectrum between $\mathrm{H} 5$ and H13/H14 protons from the methyl groups 


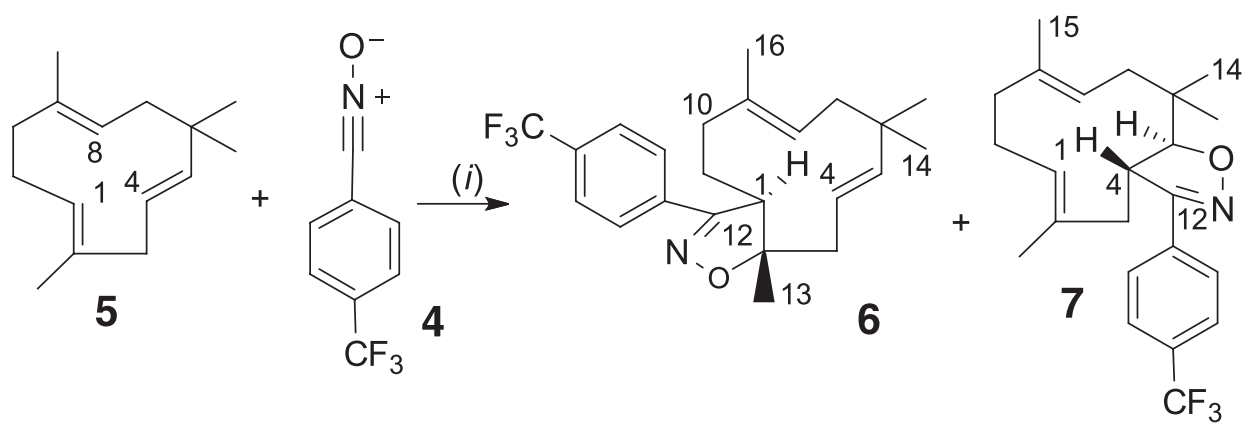

Scheme 1. Cycloaddition of dipole 4 to the dipolarophile sesquiterpene 5. (i) $\mathrm{CH}_{2} \mathrm{Cl}_{2}$, r.t. Non-systematic numbering of atoms has been used in adducts 6 and $\mathbf{7}$ for ease of comparison of spectral data.

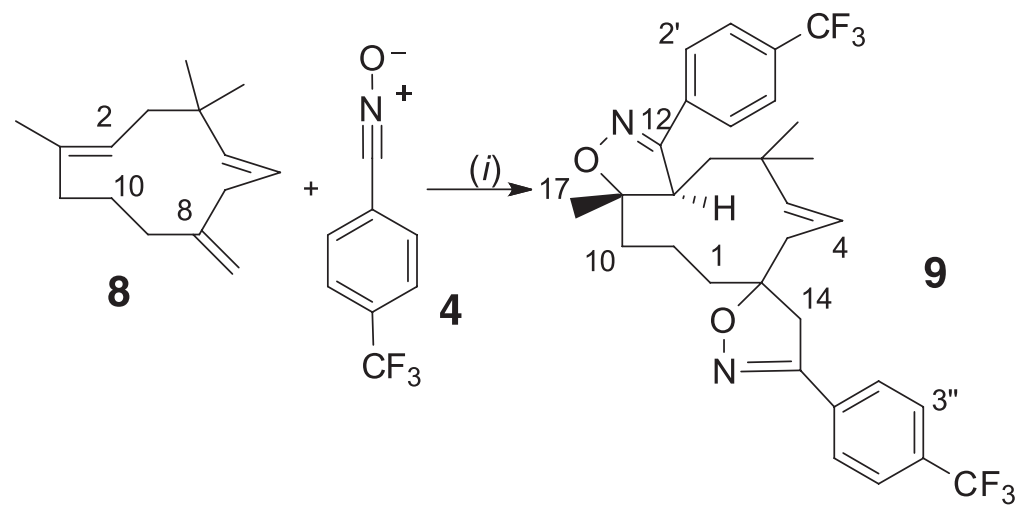

Scheme 2. Cycloaddition of the dipole 4 to the $\mathrm{C}_{15}$ sesquiterpene 8. (i) $\mathrm{CH}_{2} \mathrm{Cl}_{2}$, r.t.

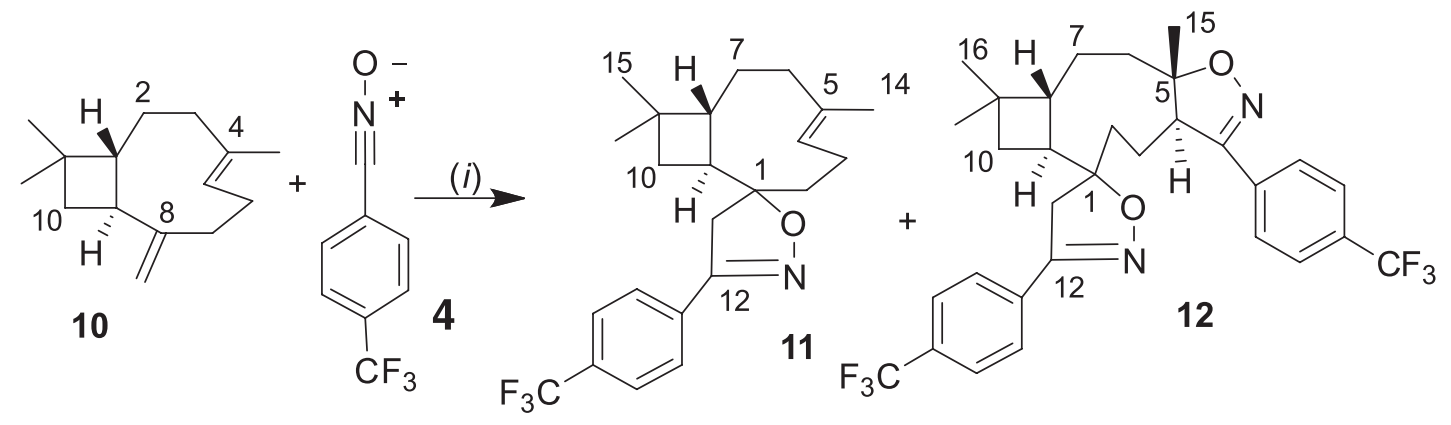

Scheme 3. Cycloaddition of the dipole 4 to bicyclic $\mathrm{C} 15$ sesquiterpene 10. (i) $\mathrm{CH}_{2} \mathrm{Cl}_{2}$, r.t. Non-systematic numbering of atoms has been used in adducts $\mathbf{1 1}$ and $\mathbf{1 2}$ for ease of comparison of spectral data.

and lack of correlations of these groups with $\mathrm{H} 4$, as well as a correlation of $\mathrm{H} 3$ with $\mathrm{C} 12$ in the $\mathrm{HMBC}$ spectrum, defined regiochemistry of the cycloaddition reaction where oxygen atom of the dipole attacked the less negative end of the olefinic bond.

Cycloaddition to the $\mathrm{C}_{15}$ sesquiterpene trans,trans8-methylene-1,4,4-trimethyl-1,5-cycloundecadiene (8) afforded a bis-adduct 9 in $55 \%$ yield (Scheme 2, Figure 1).

A complete assignment of ${ }^{1} \mathrm{H}$ and ${ }^{13} \mathrm{C}$ NMR spectra of 9 was based on analysis of $1 \mathrm{D}$ and $2 \mathrm{D}{ }^{1} \mathrm{H}$ and ${ }^{13} \mathrm{C}$ NMR spectra (COSY, HSQC and ROESY) and is presented in Experimental. Presence of $\mathrm{HC}=\mathrm{HC}$ unit in $E$ configuration (a large coupling constant of $15.5 \mathrm{~Hz}$ in ${ }^{1} \mathrm{HNMR}$ spectrum) confirmed by the 2D COSY spectrum and two olefinic $\mathrm{H}-\mathrm{C}$ correlations in HSQC spectrum indicated that $\mathrm{C} 4-\mathrm{C} 5$ double bond was preserved. Lack of H-C correlation in HSQC spectrum for carbon 9 connected to the isoxazoline oxygen atom and presence of $\mathrm{H}-\mathrm{C}$ correlation for $\mathrm{C} 8$ have shown the same regiochemistry of the reaction as in case of compound $\mathbf{6}$. Oxygen atom of the second molecule of the dipole attacked the mono-adduct exocyclic double bond at the di-substituted carbon atom as expected. The 2D HSQC spectrum showed the presence of two H14a,b-C14 (43.1 ppm) correlations. Facial selectivity was proved to be as shown in the structure 9 by observation in the 2D ROESY NMR spectrum of a proximity between $\mathrm{H} 3$ and $\mathrm{H} 14 \mathrm{a} / \mathrm{H} 14 \mathrm{~b}$.

Cycloaddition to the bicyclic $\mathrm{C} 15$ sesquiterpene trans(1R,9S)-8-methylene-4,11,11-trimethylbicyclo[7.2.0]undec- 


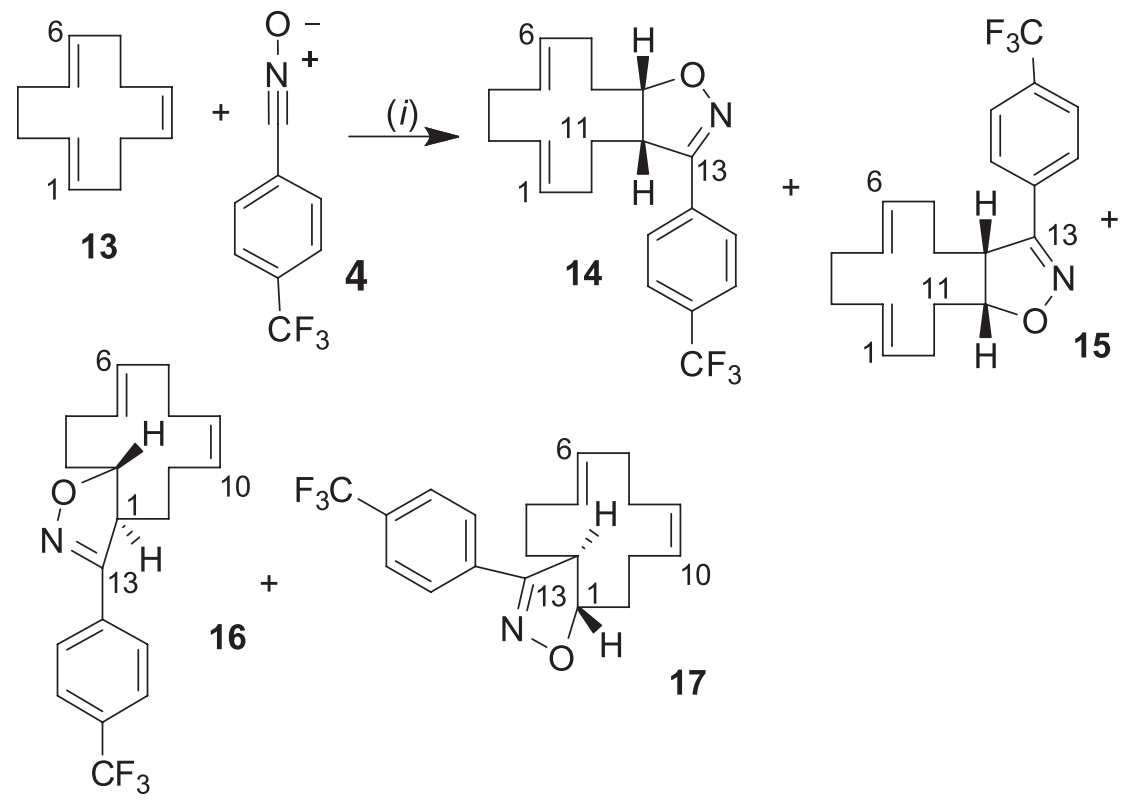

Scheme 4. Cycloaddition of the dipole 4 to trans, trans,cis-1,5,9-cyclododecatriene (13). (i) $\mathrm{CH}_{2} \mathrm{Cl}_{2}$, r.t. Non-systematic numbering of atoms has been used in adducts 14-17 for ease of comparison of spectral data.

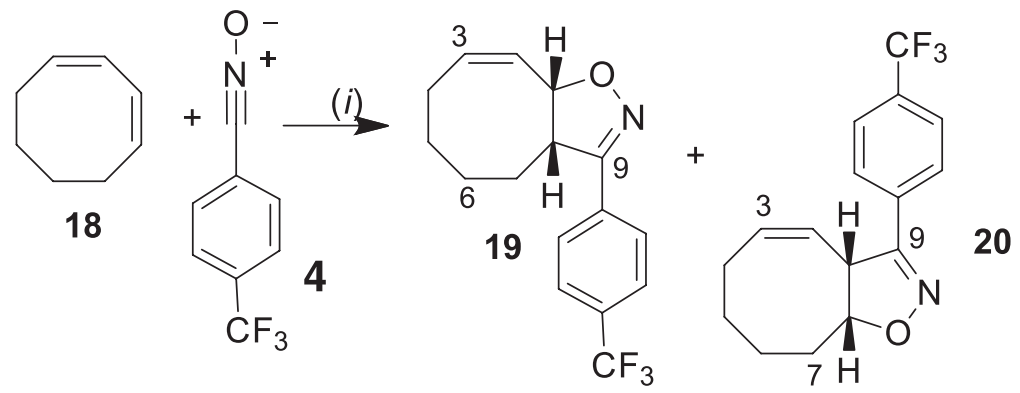

Scheme 5. Cycloaddition of the dipole 4 to 1,3-cyclooctadiene (18). (i) $\mathrm{CH}_{2} \mathrm{Cl}_{2}$, r.t. Non-systematic numbering of atoms has been used in adducts $\mathbf{1 9}$ and 20 for ease of comparison of spectral data.

4-ene (10) afforded a mixture of a mono-adduct $\mathbf{1 1}(45 \%)$ and a bis-adduct 12 (30\%) (Scheme 3, Figure 1). Structure of the mono-adduct $\mathbf{1 1}$ was established based on analysis of $1 \mathrm{D}$ and $2 \mathrm{D}{ }^{1} \mathrm{H}$ and ${ }^{13} \mathrm{C}$ NMR spectra (COSY, HSQC and ROESY); a complete assignment of ${ }^{1} \mathrm{H}$ and ${ }^{13} \mathrm{C}$ NMR spectra is presented in Experimental. Cycloaddition to the exocyclic double bond via attack of the oxygen atom of the dipole on the di-substituted carbon atom afforded a spiro system, where carbon atom $\mathrm{C} 1$ attached to oxygen did not show any H-C correlations in the 2D HSQC NMR spectrum. Facial selectivity was established by observation in the ROESY NMR spectrum of correlations between H13a,b at 3.32 and $3.19 \mathrm{ppm}$ and H10a,b at 2.07 and $1.96 \mathrm{ppm}$, as well as between $\mathrm{H} 13 \mathrm{~b}$ and aryl $\mathrm{H} 2$ '/H6' protons. The C4-C5 double bond was preserved as witnessed by observation in the ${ }^{13} \mathrm{C}$ NMR spectrum signals of the quaternary $\mathrm{C} 5$ at $133.8 \mathrm{ppm}$ and $\mathrm{C} 4$ at $120.2 \mathrm{ppm}$ coupled in the HSQC spectrum to $\mathrm{H} 4$ at $5.21 \mathrm{ppm}$.

Mass spectrometry of the other product showed molecular weight of $578 \mathrm{mu}$, indicating a bis-adduct structure 12. It showed a similar regiochemistry and geometry of the spiro isoxazoline ring as the mono-adduct 11. The 2D ROESY NMR spectrum showed a proximity of H13a (3.43) and H11 (1.92), H13b (3.20) and H10a (1.81), as well as H13a,b and aryl $\mathrm{H}^{\prime} / \mathrm{H}^{\prime}$ ' protons (7.71 ppm). Regiochemistry of the second cycloaddition step resulted from attack of the dipole oxygen atom at the more substituted carbon atom C5.

Cycloaddition to trans,trans, cis-1,5,9-cyclododecatriene (13) afforded a mixture of two inseparable regioisomeric mono-adducts to cis double bond $\mathbf{1 4}$ and $\mathbf{1 5}$ (53:47) as indicated by mass spectrometry giving a molecular weight of $349 \mathrm{mu}$, and two inseparable regioisomeric monoadducts (60:40) to the trans double bond $\mathbf{1 6}$ and $\mathbf{1 7}$ in an overall 62:38 ratio (Scheme 4). A complete assignment of ${ }^{1} \mathrm{H}$ and ${ }^{13} \mathrm{CNMR}$ spectra of the products was based on analysis of $1 \mathrm{D}$ and $2 \mathrm{D}{ }^{1} \mathrm{H}$ and ${ }^{13} \mathrm{C}$ NMR spectra (COSY, HSQC, HMBC, and ROESY NMR) and is presented in Tables 1 and 2.

Structures 14 and 15 were proposed to the major pair of adducts assuming a preferred addition to the cis-double 


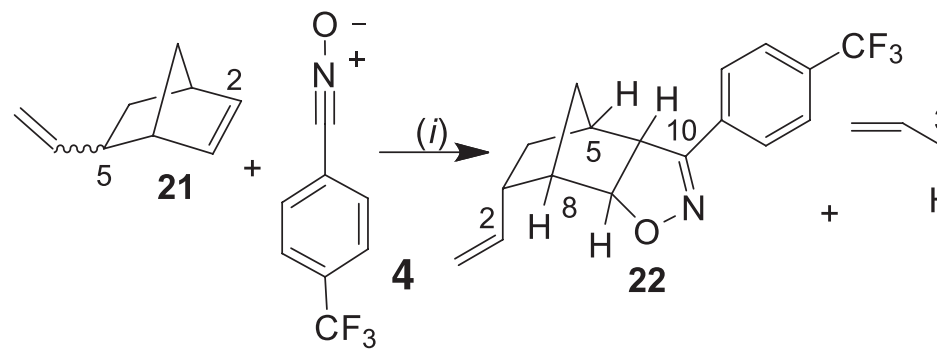

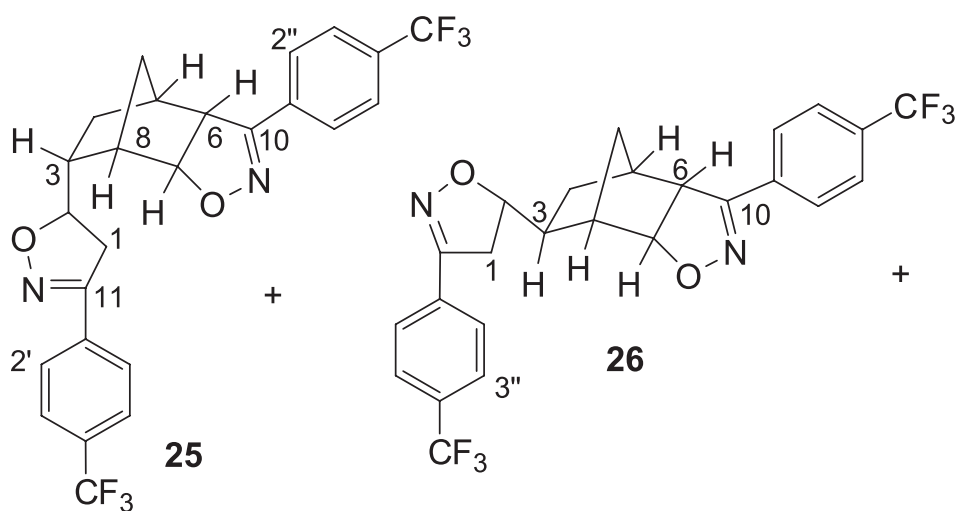

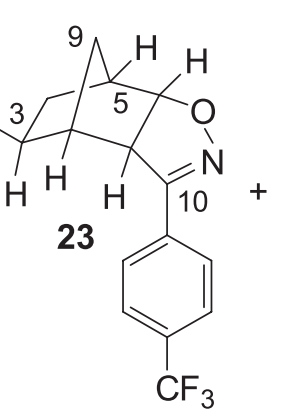

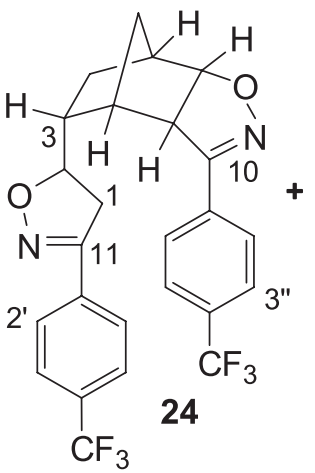

Scheme 6. Cycloaddition of the dipole 4 to 5-vinyl-2-norbornene (21). (i) $\mathrm{CH}_{2} \mathrm{Cl}_{2}$, r.t. Non-systematic numbering of atoms has been used in adducts $\mathbf{2 2}$-27

for ease of comparison of spectral data.
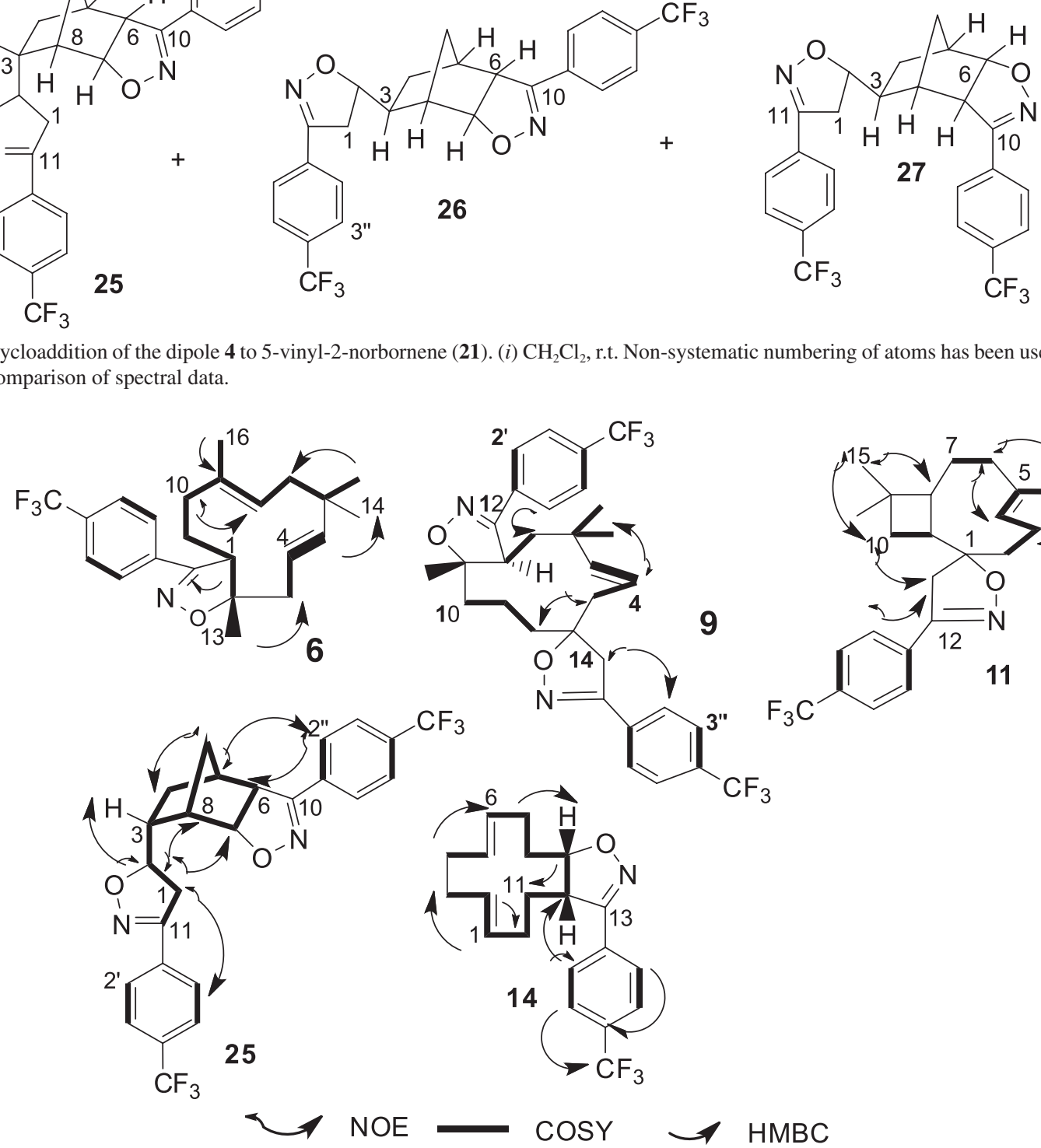

$\mathrm{HMBC}$

Figure 1. Selected COSYs, NOEs and HMBC for adducts 6, 9, 11, 14, and 25.

bond. Calculations carried out with the molecular modelling program showed lower energies for these isomers and a much smaller difference of energy between endo and exo forms than for the adducts to the trans bonds of the dipolarophile 13. Larger coupling constants
$(12.5 \mathrm{~Hz})$ were observed for the ring junction protons cis $\mathrm{H} 9 / \mathrm{H} 10$ in these regioisomers than for the corresponding trans $\mathrm{H} 1 / \mathrm{H} 2$ in isomers 16 and $\mathbf{1 7}(10.5 \mathrm{~Hz})$. Calculations carried out with HNMR Predictor software v.12 confirmed higher $J$ values for these protons in cis adducts $\mathbf{1 4}$ and 
$15(9.5 \mathrm{~Hz})$ than in trans adducts $\mathbf{1 6}$ and $\mathbf{1 7}(8.4 \mathrm{~Hz})$. Smaller values of coupling constants of the corresponding critical $\mathrm{H} 4 / \mathrm{H} 5$ protons in trans disubstituted isoxazolines than in cis disubstituted isomers were observed before. ${ }^{23}$ Structure $\mathbf{1 4}$ was proposed to the endo adduct based on the ROESY 2D NMR spectrum showing a correlation of $\mathrm{H} 10(3.20 \mathrm{ppm})$ and $\mathrm{H} 2$ ' proton $(7.80 \mathrm{ppm})$, while in the exo adduct $\mathbf{1 5}$ the corresponding proximity of $\mathrm{H} 9$ (3.41 ppm) and H2' (8.09) was absent. Model studies carried out with the Hyperchem program have confirmed this conclusion and showed spatial proximity of the relevant protons.

Similarly, endo configuration was proposed to the isomer $\mathbf{1 6}$ and exo configuration was ascribed to the isomer 17. 2D ROESY NMR spectrum of $\mathbf{1 6}$ showed a H1 (3.37 ppm)-H12a,b (1.64, $1.56 \mathrm{ppm})$ correlation, while in the other regioisomer $\mathbf{1 7}$ a corresponding correlation $\mathrm{H} 2$ (3.46 ppm)-H3a,b (1.63, 1.54 ppm) was missing.

Cycloaddition to 1,3-cyclooctadiene (18) afforded a mixture of regioisomeric mono-adducts $\mathbf{1 9}$ and $\mathbf{2 0}$ in 4:1 ratio (Scheme 5). Structure of the major isomer 19 was proposed as endo based on 2D ROESY NMR spectrum, where correlations of the bridgehead proton $\mathrm{H} 8$ at $3.74 \mathrm{ppm}$ and the aliphatic $\mathrm{H} 7$ proton at $1.75 \mathrm{ppm}$ with aromatic protons $\mathrm{H}^{\prime} / \mathrm{H} 6$ ' at $7.71 \mathrm{ppm}$ were observed, while no correlation in 2D HMBC NMR spectrum between the imine C9 carbon atom and the olefinic $\mathrm{H} 2$ proton was found, which would occur in isomer 20.

Cycloaddition to 5-vinyl-2-norbornene (21) (a mixture of exolendo epimers, 78:22) afforded mono-adducts $\mathbf{2 2}$ and $\mathbf{2 3}$ in 11:30 ratio and regioisomeric bis-adducts $\mathbf{2 4 - 2 7}$ (Scheme 6). A complete assignment of ${ }^{1} \mathrm{H}$ and ${ }^{13} \mathrm{C}$ NMR spectra of the products was based on analysis of $1 \mathrm{D}$ and $2 \mathrm{D}{ }^{1} \mathrm{H}$ and ${ }^{13} \mathrm{C}$ NMR spectra (COSY, HSQC, HMBC, and ROESY) and is presented in Tables 3 and 4 and Figure 1 for compounds 24-27. Assignments were facilitated by the published data on product of the $[2+3]$ cycloaddition of nitrile oxides to the norbornenes. ${ }^{9,24}$

In both monocycloadducts $\mathbf{2 2}$ and $\mathbf{2 3}$ reaction proceeded with a complete site-selectivity and exo-selectivity. The crucial isoxazoline $\mathrm{H} 6$ and $\mathrm{H} 7$ protons were in ${ }^{1} \mathrm{H}$ NMR spectra doublets coupled only to each other $(J 8.3-8.5 \mathrm{~Hz})$, while coupling constants with the bridgehead $\mathrm{H} 5$ and $\mathrm{H} 8$ are very small $(0-1 \mathrm{~Hz})$, which corresponds to the value of the respective dihedral angles close to $90^{\circ}$. Regiochemistry of the reaction was established by application of ${ }^{1} \mathrm{H}$ NMR and 2D NMR ROESY spectroscopy. In regioisomer 22 with syn relative position of the methylene bridge and the phenyl ring $\mathrm{H} 7$ proton at $4.65 \mathrm{ppm}$ showed a cross peak with $\mathrm{H} 8$ at $2.65 \mathrm{ppm}$, and $\mathrm{H} 6$ at $3.78 \mathrm{ppm}$ showed a cross peak with $\mathrm{H} 5$ at $2.45 \mathrm{ppm}$.
On the other hand, in regioisomer $\mathbf{2 3}$ with anti relative position of the methylene bridge and the phenyl ring, H6 proton (4.92 ppm) exhibited a cross peak with H5 (2.70 ppm), and H7 (3.51 ppm) exhibited a cross peak with $\mathrm{H} 8(2.51 \mathrm{ppm})$. Endo configuration of the vinyl side chain at $\mathrm{C} 3$ in regioisomer $\mathbf{2 2}$ was established by observance in the 2D ROESY NMR spectrum of a proximity of $\mathrm{H} 3$ (2.64) and $\mathrm{H} 9(1.57,1.37 \mathrm{ppm})$ as well as H3 and H5. It was confirmed by finding a ${ }^{4} J$ W-type coupling between H5 (2.45) and H3. On the other hand, exo configuration at C3 was proposed for the regioisomer $\mathbf{2 3}$ based on 2D ROESY NMR spectrum, where correlations H3 (2.66 ppm)-H8 (2.51) and H1 (5.12 ppm)-H4b (1.30 ppm) were observed. Presence of anti configuration was corroborated by finding a correlation C10 (156.0 ppm)-H8 (2.51 ppm) in 2D HMBC NMR spectrum.

Spectral data and elemental analyses have proved that products 24-27 are bis-adducts. The first eluted biscycloadduct $\mathbf{2 4}$ was characterized by an anti relationship of the $\mathrm{C} 10$ aryl group and the methylene bridge as proved by 2D ROESY NMR spectrum, where spatial proximity of $\mathrm{H} 6(4.80 \mathrm{ppm})$ and $\mathrm{H} 5(2.71 \mathrm{ppm})$ as well as H7 (4.20 ppm ) and H8 (2.54 ppm) was found. Correlations in 2D ROESY NMR spectrum of $\mathrm{H} 2$ (5.01 ppm)-H7 and $\mathrm{H} 2-\mathrm{H} 8$ indicate that a $\mathrm{C} 3$ substituent is in the endo position.

The second eluted bis-cycloadduct 25 showed the opposite syn relationship of the $\mathrm{C} 10$ aryl group and the methylene bridge since in the 2D ROESY NMR spectrum correlations between H6 (5.16 ppm) and H5 (2.77 ppm) as well as between $\mathrm{H} 7(3.69 \mathrm{pm})$ and $\mathrm{H} 8$ (2.56 ppm) were observed. Endo configuration at $\mathrm{C} 3$ was proposed based also on the 2D ROESY NMR results, where spatial proximity of $\mathrm{H} 1 \mathrm{~b}(3.08 \mathrm{ppm})$ and $\mathrm{H} 8, \mathrm{H} 2(4.86 \mathrm{ppm})$ and $\mathrm{H} 7$, as well as $\mathrm{H} 3(2.27 \mathrm{ppm})$ and $\mathrm{H} 9 \mathrm{~b}(1.34 \mathrm{pm})$ were found, while no correlations $\mathrm{H} 3-\mathrm{H} 7$ and $\mathrm{H} 3-\mathrm{H} 5$ were observed.

The third bis-cycloadduct $\mathbf{2 6}$ showed also syn relationship of the $\mathrm{C} 10$ aryl group and the methylene bridge because in the 2D ROESY NMR spectrum correlations between $\mathrm{H} 7$ (4.70 ppm) and $\mathrm{H} 8$ (2.70 ppm), as well as between H6 (3.99 ppm) and H5 (2.82 ppm) were observed. On the other hand, exo position of the side substituent at $\mathrm{C} 3$ was established based on correlations H1b (3.07 ppm)-H3 (2.22 ppm) and H3-H7 in the 2D ROESY NMR spectrum. This assignment was corroborated by ${ }^{13} \mathrm{C}$ NMR data, where larger chemical shift was recorded for C7 (87.9 ppm) than for the corresponding carbon atom in the endo isomer 25, $84.8 \mathrm{ppm}$ because of the gauche $\gamma$-effect.

Finally, the fourth eluted bis-cycloadduct 27 showed anti relationship of the $\mathrm{C} 10$ aryl group and the 
methylene bridge because in the 2D ROESY NMR spectrum correlations H6 (4.70 ppm)-H5 (2.70 ppm) and H7 (3.99 ppm)-H8 (2.82 ppm) were found. Exo position of the $\mathrm{C} 3$ arylisoxazoline substituent was proposed based on the proximity of $\mathrm{H} 3(2.23 \mathrm{ppm})$ and $\mathrm{H} 8$. Carbon atoms $\mathrm{C} 2$ (82.9) and $\mathrm{C} 7$ (87.9) attached to the oxygen atoms were identified by ${ }^{1} \mathrm{H}$ NMR and the 2D HSQC and HMBC spectra. $\mathrm{C} 2$ showed cross peaks with the adjacent methylene group protons at 3.53 and $3.07 \mathrm{ppm}, \mathrm{H} 3$, and $\mathrm{H} 4 \mathrm{a}$, b protons (1.90 and $0.79 \mathrm{ppm})$. On the other hand, C7 exhibited correlations with $\mathrm{H} 8, \mathrm{H} 9 \mathrm{~b}$ (1.33 ppm), and $\mathrm{H} 3$.

\section{Rationalization of the observed site selectivity and regioselectivity}

Table 5 gives electron charges at the alkenyl carbon atoms of the dipolarophiles $\mathbf{5}, \mathbf{8}, \mathbf{1 0}, \mathbf{1 3}, \mathbf{1 8}, \mathbf{2 1}$, and the observed site-selectivity in the cycloaddition reaction.

It was found that the amount of negative charges of both carbon atoms of the double bond correlated with the reactivity of the reaction. Cycloaddition to $\alpha$-humulene (5) occurred mainly to the C1-C2 double bond with the greatest amount of electric charges $(-0.303)$, and in the minor isomer the dipole added to one of the other two double bonds C4-C5 with a larger sum of electric charges $(-0.263)$. Similarly, in cycloaddition to trans caryophylene (10) in the major adduct $\mathbf{1 1}$ more reactive was an exocyclic double bond $\mathrm{C} 8-\mathrm{C} 12$ with total electric charges of -0.321 , while the other double bond had a charge of -0.273 . The same trend was observed in case of 5-vinyl-2-norbornene (21), where higher electron densities were found in the $\mathrm{C} 2-\mathrm{C} 3$ bond (-0.349/-0.342, exolendo isomers), than in the C8-C9 bond(-0.264/-0.256, exo/endo isomers). It correlated with a higher reactivity of the $\mathrm{C} 2-\mathrm{C} 3$ bond affording $41 \%$ of mono-adducts 22 and $\mathbf{2 3}$ to this bond, and 38\% of bisadducts 24-27 to C2-C3 and C8-C9 bonds.
Some discrepancy from this regularity was noticed for $\beta$-humulene (8), where the bis-adduct 9 was formed in reaction of the most electron-rich $\mathrm{C} 8-\mathrm{C} 12$ bond and the least electron rich $\mathrm{C} 1-\mathrm{C} 2$ double bond. It could be explained by the steric factors and a more difficult approach of the dipole to the hindered internal double bond of the dipolarophile flanked with two geminal methyl groups.

\section{Biological activity of the products}

Generally, only a modest or weak activity of the compounds synthesized in this project was recorded (Table 6). The highest fungicidal inhibitory potency was found against Botrytis cinerea strain of mono cycloadducts 6 and 19-20 pair. It was comparable to the activity of the reference compound, the commercial fungicide chlorothalonil.

Analysis of the data from Table 6 allows concluding that generally exo regioisomers show a higher activity than the endo regioisomers. As an example isomer $\mathbf{6}$ was more active than isomer $\mathbf{7}$, and compounds $\mathbf{2 2}$ and $\mathbf{2 5}$ were more active than, respectively, cycloadducts $\mathbf{2 3}$ and $\mathbf{2 4}$. One can see very clearly the influence of the spatial structure on the fungicidal activity.

\section{Conclusions}

High regio- and site selectivity of [2+3] dipolar cycloaddition reaction of 4-trifluoromethylbenzonitrile oxide (4) to sesquiterpene 8 were observed. A correlation of double bond reactivity with electron charges was found in reactions of most dipolarophiles. The addition to the exocyclic double bond was favored. All diastereoisomeric products were fully characterized by ${ }^{1} \mathrm{H}$ and ${ }^{13} \mathrm{C}$ NMR 1D and 2D spectroscopy. Some cycloadducts showed good fungistatic activity. Further research is in progress to analyze the biological potency of the new products, and to improve regioselectivity of the cycloaddition.

Table 5. Electric charges at the alkenyl carbon atoms of the dipolarophiles $\mathbf{5}, \mathbf{8}, \mathbf{1 0}, \mathbf{1 3}, \mathbf{1 8}, \mathbf{2 1}$ and site-selectivity in the cycloaddition reaction

\begin{tabular}{|c|c|c|c|c|c|c|c|c|}
\hline \multirow{2}{*}{ Dipolarophile/C atom } & \multicolumn{8}{|c|}{ Electric charge } \\
\hline & $\mathrm{C} 1$ & $\mathrm{C} 2$ & $\mathrm{C} 4 / 6$ & $\mathrm{C} 3 / \mathrm{C} 5$ & $\mathrm{C} 8$ & $\mathrm{C} 9 / \mathrm{C} 12$ & $\mathrm{C} 10$ & Site selectivity \\
\hline 5 & -0.16 & -0.11 & -0.16 & -0.144 & -0.154 & -0.106 & - & $\mathrm{C} 1 / \mathrm{C} 2$ \\
\hline 8 & -0.106 & -0.159 & -0.152 & -0.154 & -0.093 & -0.234 & -0.152 & $\mathrm{C} 1 / \mathrm{C} 2$ \\
\hline 10 & - & - & -0.104 & -0.169 & -0.086 & -0.235 & - & $\mathrm{C} 8 / \mathrm{C} 12$ \\
\hline 13 & -0.159 & -0.159 & -0.159 & -0.159 & - & -0.159 & -0.159 & C9/C10 \\
\hline 18 & -0.150 & -0.141 & -0.150 & -0.141 & - & - & - & - \\
\hline 21 exo & - & -0.173 & - & -0.176 & -0.151 & -0.113 & - & $\mathrm{C} 2 / \mathrm{C} 3$ \\
\hline 21endo & - & -0.172 & - & -0.170 & -0.145 & -0.111 & - & $\mathrm{C} 2 / \mathrm{C} 3$ \\
\hline
\end{tabular}


Table 6. Fungicidal inhibitory activities of compounds $6,7,11,12,14,15,16,17,19,20,22,23,24,25,26,27$ at $200 \mathrm{mg} \mathrm{L}^{-1}$

\begin{tabular}{|c|c|c|c|c|c|}
\hline \multirow{2}{*}{ Compound } & \multicolumn{5}{|c|}{ Linear growth inhibition / \% } \\
\hline & A. $a$. & B. $c$. & F. $c$. & P. c. & Rh. s. \\
\hline 6 & 0 & 78.7 & 24.0 & 2.4 & 0 \\
\hline 7 & 10 & 0 & 0 & 0 & 11 \\
\hline 11 & 0 & 15.6 & 0 & 11.1 & 0 \\
\hline 12 & 13 & 0 & 4.4 & 0 & 0 \\
\hline $19+20$ & - & 73.0 & 38.0 & 12.0 & 11.0 \\
\hline $14+15$ & 17.4 & 20.0 & 0 & 11.1 & 30.0 \\
\hline $16+17$ & 10.9 & 28.9 & 0 & 37.8 & 18.0 \\
\hline 22 & 26.0 & 28.0 & 0 & 0 & 24.0 \\
\hline 23 & 13.0 & 4.4 & 0 & 11.1 & 0 \\
\hline 24 & 4.3 & 8.9 & 11.1 & 17.8 & 0 \\
\hline 25 & 15.2 & 31.1 & 0 & 22.2 & 0 \\
\hline 26 & 0 & 11.1 & 4.4 & 4.4 & 0 \\
\hline 27 & 0 & 8.9 & 0 & 15.6 & 0 \\
\hline Chlorothalonil $^{\text {a }}$ & - & 80.0 & 38.0 & 61.0 & 88.0 \\
\hline
\end{tabular}

${ }^{a}$ Reference compound. A. a.: Alternaria alternate; B. c.: Botrytis cinerea; F. c.: Fusarium culmorum; P. c.: Phytophtora cactorum; Rh. s.: Rhizoctonia solani.

\section{Supplementary Information}

Supplementary data $\left({ }^{1} \mathrm{H}\right.$ ROESY, COSY, ${ }^{1} \mathrm{H}^{-}{ }^{13} \mathrm{C} \mathrm{HSQC}$, ${ }^{1} \mathrm{H}-{ }^{13} \mathrm{C}$ HMBC NMR spectra) are available free of charge at http://jbcs.sbq.org.br as PDF file.

\section{Acknowledgments}

This work was supported in part by the Polish Ministry of Science and Higher Education (Research Grant EMC 902000031), which is gratefully acknowledged.

\section{References}

1. Bast, K.; Christl, M.; Huisgen, R.; Mack, W.; Sustman, R.; Chem. Ber. 1973, 106, 3258.

2. Caramella, P.; Grunanger, P. In 1,3-Dipolar Cycloaddition Chemistry; Padwa, A., ed.; Wiley: New York, 1984, pp. 177.

3. Mukayama, T.; Hoshino, T.; J. Am. Chem. Soc. 1960, 82, 5339.

4. Shimizu, T.; Hayashi, T.; Shibafuchi, H.; Teramura, K.; Bull. Chem. Soc. Jpn. 1986, 59, 2827.

5. Gothelf, K. V.; Jørgensen, K. A.; Chem. Rev. 1998, 98, 863; Pellisier, H.; Tetrahedron 2007, 63, 3235; Kissane, M.; Maguire, A. R.; Chem. Soc. Rev. 2010, 39, 845.

6. Kanemasa, S.; Nishiuchi, M.; Kamimura, A.; Hori, K.; J. Am. Chem. Soc. 1994, 116, 2324.

7. Fisera, L.; Ondrus, V.; Timpe, H. J.; Collect. Czech. Chem. Commun. 1990, 55, 512.

8. Gucma, M.; Gołębiewski, W. M.; Krawczyk, M.; J. Braz. Chem. Soc. 2013, 24, 805.
9. Gucma, M.; Gołębiewski, W. M.; Michalczyk, A. K.; J. Mol. Struct. 2014, 1060, 223.

10. Coutouli-Argyropoulou, E.; Anastasopoulos, C.; J. Heterocycl. Chem. 1996, 33, 731.

11. Baldwin, J. E.; Mayweg, A. V. W.; Neumann, K.; Pritchard, G. J.; Org. Lett. 1999, 1933.

12. Zhang, Y.-H.; Itoh, K.; Gao, D.; Chang, Y.; Cheng, J.-P.; Chow, Y. L.; Res. Chem. Intermed. 2002, 28, 313.

13. Chow, Y. L.; Wang, S.-S.; Johansson, C. I.; Liu, Z.-L.; J. Am. Chem. Soc. 1996, 118, 11725.

14. Nakayama, J.; Tajima, Y.; Xue-hua, P.; Sugihara, Y.; J. Am. Chem. Soc. 2007, 129, 7250.

15. Mach, K.; Antropiusová, H.; Petrusová, L.; Hanuš, V.; Tureček, F.; Sedmera, P.; Tetrahedron 1984, 40, 3295.

16. Moriconi, E. J.; Hummel, C. F. J.; J. Org. Chem. 1976, 41, 3583.

17. Nozaki, H.; Kurita, M.; Mori, T.; Noyori, R.; Tetrahedron 1968 , 24, 1821.

18. Bak, D. A.; Brady, W. T. J.; J. Org. Chem. 1979, 44, 107.

19. Foley, A. D.; Maguire, A. R.; Tetrahedron 2010, 66, 1131.

20. Kikuchi, D.; Yoshida, M.; Shishido, K.; Synlett 2012, 4, 577.

21. Liu, K. C.; Shelton, B. R.; Howe, R. K.; J. Org. Chem. 1980, $45,3916$.

22. Hyperchem 7.5; Hypercube, Inc., Gainesville, USA, 2003.

23. Kanemasa, S.; Hayashi, T.; Yamamoto, H.; Wada, E.; Sakurai, T.; Bull. Chem. Soc. Jpn. 1991, 64, 3274.

24. Mayo, P.; Hecnar, T.; Tam, W.; Tetrahedron 2001, 57, 5931.

Submitted: January 28, 2016 Published online: March 16, 2016 